TRANSACTIONS OF THE

AMERICAN MATHEMATICAL SOCIETY

Volume 363, Number 3, March 2011, Pages 1487-1508

S 0002-9947(2010)05129-3

Article electronically published on October 15, 2010

\title{
MULTI-DIMENSIONAL MORSE INDEX THEOREMS AND A SYMPLECTIC VIEW OF ELLIPTIC BOUNDARY VALUE PROBLEMS
}

\author{
JIAN DENG AND CHRISTOPHER JONES
}

\begin{abstract}
Morse Index Theorems for elliptic boundary value problems in multi-dimensions are proved under various boundary conditions. The theorems work for star-shaped domains and are based on a new idea of measuring the "oscillation" of the trace of the set of solutions on a shrinking boundary. The oscillation is measured by formulating a Maslov index in an appropriate Sobolev space of functions on this boundary. A fundamental difference between the cases of Dirichlet and Neumann boundary conditions is exposed through a monotonicity that holds only in the former case.
\end{abstract}

\section{INTRODUCTION}

The problem of determining the Morse index (number of unstable eigenvalues), an algebraic object, from the geometric structure of the underlying solution in question is an old one. In one space dimension, Sturm-Liouville theory affords a direct relationship between the Morse index and the number of nodes of the solution. The Morse Index Theorem ([17]) states that the Morse index can be read off from the number of conjugate points along a geodesic, and this can be generalized to Hamiltonian systems of any finite dimension. This affords a symplectic interpretation (see Arnol'd [1, 2]). From this perspective, conjugate points are related to the location where Lagrangian planes of the Hamiltonian system have nontrivial intersection. This, in turn, corresponds to the "oscillation" of the Lagrangian plane in the total Lagrangian Grassmannian which is given by the Maslov index. The equality of the Morse index with the Maslov index thus offers a way of gleaning algebraic information (Morse index) from geometric information (Maslov index). This notion of oscillation also illustrates that the Morse Index Theorem is really a natural generalization of the Sturm-Liouville theory of oscillations for second-order scalar ODE's.

To generalize the Morse Index Theorem to the case of domains in higher space dimensions, Smale (18]) studied elliptic operators on smooth manifolds with boundary. In that work, Smale introduced the idea of shrinking the manifold to a small set (in the sense of measure he assumes that the space possesses). He then generalizes the notion of conjugate points in a natural way to this case. The Morse Index Theorem can be reformulated and still holds. Later, Swanson ([19]) developed an infinite-dimensional Lagrangian intersection theory to reinterpret Smale's result.

Received by the editors July 3, 2008 and, in revised form, June 8, 2009.

2000 Mathematics Subject Classification. Primary 35J25, 35P15; Secondary 53D12, 35B05.

Key words and phrases. Morse index, Maslov index, elliptic boundary value problem.

(C) 2010 American Mathematical Society Reverts to public domain 28 years from publication 
It is natural to think of this problem in the context of the effort to relate the index for the eigenvalue problem (number of eigenvalues greater than, for instance, zero) of a selfadjoint operator, arising from a linear elliptic equation posed on a multi-dimensional domain. There is a long history of work aimed at relating this index to the nodal structure of the associated eigenfunction. This goes back, at least to Courant and Hilbert [3], and was further developed in [9] and [11]. The interesting paper of Johnson, see [13], can also be seen in this light.

In this paper, we offer a new way of looking at this problem that rests on the dynamical systems interpretation of Sturm-Liouville theory and the Morse Index Theorem in terms of oscillations. The key step is to set the elliptic problem in a symplectic context and use the Maslov index to relate the Morse index to a geometric construction that encodes a generalized notion of oscillation. This is similar in spirit to the work of Swanson [19], but the construction is different in a critical respect and we introduce a fundamentally new approach which we believe will be of broader applicability.

The key new idea in our work is to introduce a "sweeping" of the underlying spatial domain by shrinking the boundary. The traces of all the solutions to the elliptic problem (without any boundary conditions) restricted to a shrinking boundary is then realized in an appropriate Grassmannian of Lagrangian planes of a symplectic Hilbert space. The "oscillation" of this curve, i.e., its Maslov index, is then related to the Morse index. By realizing the boundary conditions as an appropriate subset of this space, the interpretation of the Maslov index in terms of "conjugate points" can then be made in a natural way.

The contrast of this approach with that of Smale shows how we are able to obtain results that go beyond those in Smale's paper. There are two different approaches to the Morse Index Theorem. The original approach, see, for instance, Milnor [17, uses a variational method. This works well in this context with Dirichlet boundary conditions, which Smale assumes. The approach using the Maslov index, (see [2]), is more general, and this is revealed in this framework by its ability to handle more general boundary conditions including the important Neumann case. Within the framework of the Maslov index, the difference can be seen clearly, as a certain monotonicity holds for Dirichlet boundary conditions but not for the Neumann case. The implication is that a different type of Morse Index Theorem holds in this case.

\section{FRAMEWORK AND RESULTS}

The basic setup will be of a semilinear elliptic boundary value problem:

$$
\begin{gathered}
-\Delta u+g(x, u)=0, \quad x \in \Omega, \\
P\left(\left.u\right|_{\partial \Omega},\left.\frac{\partial u}{\partial n}\right|_{\partial \Omega}\right)=0,
\end{gathered}
$$

where $\Omega \subset R^{m}$ is a star-shaped, bounded domain with smooth boundary, $m \geq 1$, and $n=\mathbf{n}(x)$ is the outer normal direction for $x \in \partial \Omega$. We assume that $g(x, u) \in$ $C^{3}(\bar{\Omega} \times R)$.

Equation (2.2) is to prescribe the boundary condition via a projection operator $P$. The usual Dirichlet and Neumann boundary conditions are given by projections onto the first and second coordinates respectively. Conditions on the operator $P$, which will include these two cases, will be specified later. 
We shall assume that there exists a $C^{2}$ solution $\bar{u}(x)$ of the boundary value problem (2.1)-(2.2). The existence and regularity problem for the semilinear elliptic boundary value problems has been studied extensively; see, for example, 4], [16, [10] and the references therein. For the (classical) solution $\bar{u} \in C^{2}(\bar{\Omega})$, the Morse index $M(\bar{u})$ is defined via the eigenvalue problem for the linearization at $\bar{u}$ :

$$
\begin{gathered}
A w \stackrel{\text { def }}{=}-\Delta w+f(x) w=\lambda w, \quad w \in H^{1}(\Omega), \\
P\left(\left.w\right|_{\partial \Omega},\left.\frac{\partial w}{\partial n}\right|_{\partial \Omega}\right)=0
\end{gathered}
$$

where $f(x) \stackrel{\text { def }}{=} g_{u}(x, \bar{u}(x))$. The second-order elliptic operator $A$, when restricted to $H_{P} \stackrel{\text { def }}{=}\left\{w \in H^{2}(\Omega) \mid P\left(\left.w\right|_{\partial \Omega},\left.\frac{\partial w}{\partial n}\right|_{\partial \Omega}\right)=0\right\}$, will be a selfadjoint operator with compact resolvent, and the Morse index $M(\bar{u})$ is defined as the dimension of the maximal subspace of $H_{P}$ on which $A$ is negative-definite.

The Morse index contains information about the dynamics near the solution $\bar{u}$ when it is considered as a steady state of an appropriate evolution equation. For instance, if the dynamics come from the gradient flow in $L^{2}$, the evolution equation is the associated reaction-diffusion equation

$$
u_{t}=\triangle u+g(x, u)
$$

with the same boundary conditions (2). In this case, the Morse index is the number of unstable directions at $\bar{u}$. In general, the study of the Morse index appears naturally in the finite and infinite-dimensional Morse theory and the global analysis of the calculus of variations; see [17, [4, [16] and the references therein.

To put the study of the Morse index in a symplectic context, we shall use the Hilbert space $H \stackrel{\text { def }}{=} H^{\frac{1}{2}}(\partial \Omega) \times H^{-\frac{1}{2}}(\partial \Omega)$. This will be viewed as a phase space in a dynamical sense. The space $H$ carries a natural symplectic structure $\omega$ on $H$ given by:

$$
\omega\left(\left(v_{1}, s_{1}\right),\left(v_{2}, s_{2}\right)\right)=\left\langle v_{1}, s_{2}\right\rangle-\left\langle v_{2}, s_{1}\right\rangle,
$$

where $\left(v_{1}, s_{1}\right),\left(v_{2}, s_{2}\right) \in H$, with $\langle$,$\rangle being the pairing between H^{\frac{1}{2}}(\partial \Omega)$ and $H^{-\frac{1}{2}}(\partial \Omega)$; see [14].

Denoting the set of all weak solutions $w \in H^{1}(\Omega)$ which satisfy (2.3) as $K_{\lambda}$, we note that the well-known Green's formula,

$$
\iint_{\Omega} \Delta v \cdot w-\triangle w \cdot v \quad d x=\int_{\partial \Omega} \frac{\partial v}{\partial n} \cdot w-\frac{\partial w}{\partial n} \cdot v d y
$$

holds for any $w \in K_{\lambda}, v \in C^{\infty}(\bar{\Omega})$. A key observation is that it offers a connection between the set of weak solutions $w$ of (2.3) in $K_{\lambda}$ and their boundary information $\left(\left.w\right|_{\partial \Omega},\left.\frac{\partial w}{\partial n}\right|_{\partial \Omega}\right)$ in the phase space $H$. This also prompts the following trace map on $K_{\lambda}$ via

$$
T(w)=\left(\left.w\right|_{\partial \Omega},\left.\frac{\partial w}{\partial n}\right|_{\partial \Omega}\right), \quad w \in K_{\lambda} .
$$

We should caution that no boundary conditions are imposed on functions in $K_{\lambda}$. Using the Green's formula (2.5), we easily see that the symplectic form $\omega$ vanishes on $T\left(K_{\lambda}\right)$. The symplectic form will also vanish on ker $P$. (This latter point is easy to see if $P$ is a projection onto one of the two coordinates, i.e., for Dirichlet or Neumann conditions. We shall set below conditions on $P$ for which this is more generally true.) 
A key point is then that these two subspaces, $T\left(K_{\lambda}\right)$ and ker $P$, are Lagrangian subspaces of the symplectic Hilbert space $H$. A Lagrangian subspace $L$ in a symplectic Hilbert space $H$ is a maximal closed subspace in $H$ on which $\omega$ vanishes, i.e., $\omega(p, q)=0$ for all $p, q \in L$.

To ensure that the kernel of $P$ is Lagrangian, we give the following.

Definition 2.1. The bounded linear operator $P: H \rightarrow H$ is called a Lagrangian projection operator if it satisfies $P^{2}=P$, and that the kernel ker $P \stackrel{\text { def }}{=} P^{-1}(0)$ and the range $P(H) \subset H$ are both Lagrangian subspaces of $H$.

The possible Lagrangian boundary conditions will be further divided into two types, one of which is like a Dirichlet condition and the other of which has greater affinity with Neumann conditions.

Definition 2.2. Suppose that $L$ is a Lagrangian subspace of $H$. Then it is said to be

(1) Dirichlet-based if $L=\left\{\left(S^{\prime} v, v\right) \mid v \in H^{-\frac{1}{2}}(\partial \Omega)\right\}$, where $S^{\prime}: H^{-\frac{1}{2}}(\partial \Omega) \rightarrow$ $H^{\frac{1}{2}}(\partial \Omega)$ is compact, and $\left(S^{\prime}\right)^{*}=S^{\prime}$. A Dirichlet-based Lagrangian subspace $L$ is said to be nonpositive if $S^{\prime} \leq 0$, or

(2) Neumann-based if $L=\left\{(u, S u) \mid u \in H^{\frac{1}{2}}(\partial \Omega)\right\}$, where $S: H^{\frac{1}{2}}(\partial \Omega) \rightarrow$ $H^{-\frac{1}{2}}(\partial \Omega)$ is compact, and $S^{*}=S$.

Here by saying that $S^{\prime}: H^{-\frac{1}{2}}(\partial \Omega) \rightarrow H^{\frac{1}{2}}(\partial \Omega)$ satisfies $\left(S^{\prime}\right)^{*}=S^{\prime}$, we mean that $\left\langle S^{\prime} u, v\right\rangle=\left\langle S^{\prime} v, u\right\rangle$, for all $u, v \in H^{-\frac{1}{2}}(\partial \Omega)$, where $\langle$,$\rangle is the natural pairing$ between $H^{\frac{1}{2}}(\partial \Omega)$ and $H^{-\frac{1}{2}}(\partial \Omega)$. A similar explanation can be made for $S^{*}=S$.

We assume that

(Ha) The bounded operator $P$ is a Lagrangian projection, and the kernel ker $P$ is either Dirichlet-based or Neumann-based.

Remark 2.3. Our result also holds for more general boundary conditions, such as ker $P=\left\{(u,(B+S) u) \mid u \in H^{\frac{1}{2}}(\partial \Omega)\right\}$, where $B, S: H^{\frac{1}{2}}(\partial \Omega) \rightarrow H^{-\frac{1}{2}}(\partial \Omega)$ are bounded operators, $B<\mathbf{N}$, where $\mathbf{N}: H^{\frac{1}{2}}(\partial \Omega) \rightarrow H^{-\frac{1}{2}}(\partial \Omega)$ is the standard Dirichlet-to-Neumann operator [20], $S$ is compact relative to $B$, and $B^{*}=B, S^{*}=$ $S$. We will nevertheless restrict ourselves to the simpler assumption (Ha) above for the sake of clarity.

The two fundamental Lagrangian projections $P_{D}(u, v) \stackrel{\text { def }}{=}(u, 0), \quad P_{N}(u, v) \stackrel{\text { def }}{=}$ $(0, v)$, for $(u, v) \in H$, correspond to the Dirichlet and Neumann boundary conditions, respectively, and they both belong to the class of Lagrangian projections we consider here. In this way the existence problem for (2.3) and (2.4) is reduced to that of a (nontrivial) Lagrangian intersection between $T\left(K_{\lambda}\right)$ and $\operatorname{ker} P$.

The Maslov index describes the "winding effect" of an (at least) continuous path of Lagrangian subspaces in the totality of the so-called Fredholm Lagrangian Grassmannian of $H$. To introduce the Maslov index, we first set up some notation. For a real (separable) symplectic Hilbert space $(H, \omega)$, denote by $\Lambda(H)$, the totality of all Lagrangian subspaces of $H$, which is called the Lagrangian Grassmannian. For $\mu, \eta \in \Lambda(H)$, the pair $(\mu, \eta)$ is called a Fredholm pair if $\operatorname{dim}(\mu \cap \eta)<+\infty$, and $\mu+\eta$ is closed and of finite codimension. The Fredholm Lagrangian Grassmannian with respect to $\mu \in \Lambda(H)$ is given by $F \Lambda_{\mu}(H)=\{\eta \in \Lambda(H) \mid(\mu, \eta)$ is a Fredholm pair $\}$.

The definition and properties of the Fredholm Lagrangian Grassmannian have been studied extensively in the article of Furutani [14; here we use the notation 
in that paper as much as possible. For a continuous path $\gamma:[0,1] \rightarrow F \Lambda_{\mu}(H)$, one can assign a geometric quantity, the so-called Maslov index $\mathbf{M} a s(\{\gamma(t)\}, \mu)$, as given in [14.

It will be helpful to fix $\operatorname{ker} P$ as $\mu$, the Lagrangian subspace corresponding to the boundary condition, and our goal is to first show that $T\left(K_{\lambda}\right)$, the trace map applied to the set of the weak solutions for (2.3), actually lives in $F \Lambda_{\mu}(H)$, the Fredholm Lagrangian Grassmannian with respect to $\mu$. Secondly, we will develop a dynamical system approach of constructing an (at least) continuous path $\gamma$ in $F \Lambda_{\mu}(H)$, so that the Maslov index $\mathbf{M} a s(\{\gamma(t)\}, \mu)$ will give us the Morse index $M(\bar{u})$. The idea is what we call the "domain shrinking" method and is described below.

By assumption the domain $\Omega$ is star-shaped with respect to some inner point $x_{0} \in \Omega$; therefore for each $x \in \Omega, x \neq x_{0}$, there is a unique $0<t<1, y \in$ $\partial \Omega$ such that $x-x_{0}=t y$. Without loss of generality, let $x_{0}=0$. Let $\Omega_{t} \stackrel{\text { def }}{=}$ $\{x \in \Omega: x=s y, 0 \leq s<t, y \in \partial \Omega\}, t \in(0,1]$, and $H_{t} \stackrel{\text { def }}{=} H^{\frac{1}{2}}\left(\partial \Omega_{t}\right) \times H^{-\frac{1}{2}}\left(\partial \Omega_{t}\right)$, equipped with the same symplectic form $\omega$ as that for $H$. It will be shown (see Lemma 4.4) that for each $t \in(0,1], \tilde{\Phi}_{t}:\left(H_{t}, \omega\right) \rightarrow\left(H, t^{m-1} \omega\right)$, given by

$$
\tilde{\Phi}_{t}(u(x), v(x))=(\tilde{u}(y), \tilde{v}(y)),
$$

is symplectic, where $\tilde{u}(y)=u(t y), \tilde{v}(y)=v(t y)$.

For each $t \in(0,1]$, denote by $K_{\lambda, t}$ the set of $H^{1}$ weak solutions of (2.3) in $\Omega_{t}, \lambda \in R$. We use the following trace map $T_{t}: K_{\lambda, t} \rightarrow H_{t}$ :

$$
T_{t}(w) \stackrel{\text { def }}{=}\left(\left.w\right|_{\partial \Omega_{t}},\left.\frac{\partial w}{\partial n}\right|_{\partial \Omega_{t}}\right), w \in K_{\lambda, t} .
$$

In each "shrunken phase space" $H_{t}$ we now have a Lagrangian subspace $T_{t}\left(K_{\lambda, t}\right)$. To see the "winding effect" of these Lagrangian subspaces, we need to map them into a single symplectic Hilbert space, which we choose to be $H$. The mapping needed is the natural scaling map $\Phi_{t}$ between $\partial \Omega_{t}$ and $\partial \Omega$, from which the induced mapping $\tilde{\Phi}_{t}$ will map $T_{t}\left(K_{\lambda, t}\right)$ into the corresponding Lagrangian subspace in $H$. The desired curve $\gamma$ in the Lagrangian Grassmannian $F \Lambda_{\mu}(H)$ can then be defined by

$$
\gamma:(0,1] \rightarrow F \Lambda_{\mu}(H), \quad \gamma(t) \stackrel{\text { def }}{=} \tilde{\Phi}_{t} \circ T_{t}\left(K_{0, t}\right), \quad t \in(0,1] .
$$

Due to the standard regularity result for weak solutions of (2.3), we will show that $\gamma \in C^{2}\left((0,1] ; F \Lambda_{\mu}(H)\right)$. This curve describes the evolution of Lagrangian subspaces of weak solutions for (2.3) as $t$ increases, where $\lambda=0$ is fixed.

The reason why we fix $\lambda=0$ in the construction of the curve $\gamma$ can be seen from classical Sturm-Liouville theory, where one distinct characteristic is that the information about the Morse index of the second-order selfadjoint differential operator is encoded in the behavior of the eigenfunctions at $\lambda=0$. In the same spirit, we want to show that the Morse index $M(\bar{u})$ is also encoded in the behavior of the weak solutions of (2.3), (2.4), by reading off the "winding effect" of the Lagrangian curve $\gamma$ in $F \Lambda_{\mu}(H)$ when $\lambda$ is fixed to be 0 in (2.3).

Our main results are the following.

Theorem 2.4 (Dirichlet-based case). Assuming (Ha), and that $\mu=\operatorname{ker} P$ is a nonpositive Dirichlet-based Lagrangian subspace of $H$, let $\bar{u} \in C^{2}(\bar{\Omega})$ be a solution 
for (2.1) and (2.2), and $f(0)=g_{u}(0, \bar{u}(0))>0$. Then

$$
M(\bar{u})=-\mathbf{M} a s\left(\left.\{\gamma(t)\}\right|_{[\delta, 1]}, \mu\right)
$$

for $\delta>0$ sufficiently small.

Theorem 2.5 (Neumann-based case). Assuming (Ha), and that $\mu=\operatorname{ker} P$ is a Neumann-based Lagrangian subspace of $H$, let $\bar{u} \in C^{2}(\bar{\Omega})$ be a solution for (2.1) and (2.2), $f(0)=g_{u}(0, \bar{u}(0))$. Then

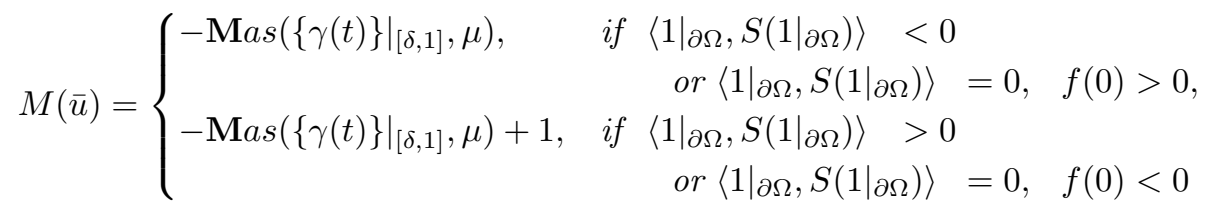

for $\delta>0$ sufficiently small.

The key idea in the proofs of Theorems 2.4 and 2.5 is the monotonicity of the Maslov index with respect to the eigenvalue parameter $\lambda \in R$.

As an application of Theorem 2.4, we discuss the monotonicity of the eigenvalue of operator $A$ with respect to the domain, for both Dirichlet and Neumann boundary conditions. We show that there is an essential difference between Dirichlet and Neumann boundary conditions, as far as the monotonicity of the eigenvalue of $A$ with respect to the domain is concerned. As given in 21, the monotonicity of the eigenvalue of a "pure" Laplacian for both Dirichlet and Neumann boundary conditions has been established 11 but for a general second-order elliptic operator $A$, the monotonicity of the eigenvalue with respect to the domain is not true for the Neumann boundary condition, though it is true for the Dirichlet boundary conditions always.

Corollary 2.6 (Dirichlet case). Assuming (Ha), let $\bar{u} \in C^{1}(\bar{\Omega})$ be a solution for (2.1) and (2.2), $f(x)=g_{u}(x, \bar{u}(x))$. For each $t \in(0,1]$, let $c(t)$ be the dimension of the solution sets for

$$
\begin{gathered}
-\triangle w+f(x) w=0, \quad \text { in } \Omega_{t}, \\
w=0, \quad \text { for } \quad x \in \partial \Omega_{t} .
\end{gathered}
$$

Then we have $M(\bar{u})=\sum_{0<t<1} c(t)$.

As a consequence of Corollary 2.6, the set of $t$ where $c(t) \neq 0$ is discrete.

Corollary 2.7 (Neumann case). Assuming (Ha), let $\bar{u} \in C^{2}(\bar{\Omega})$ be a solution for (2.1) and (2.2), $f(x)=g_{u}(x, \bar{u}(x))$, and assume that the dimension of the solution sets for

$$
\begin{gathered}
-\triangle w+f(x) w=0, \quad \text { in } \Omega, \\
\frac{\partial w}{\partial n}=0, \quad \text { for } \quad x \in \partial \Omega,
\end{gathered}
$$

is 1 , spanned by a nontrivial solution $\bar{w}(x)$. Denote the Morse index of $A=-\triangle+$ $f(x)$ restricted to $\Omega_{t}$ under the Neumann boundary condition as $M(t), t \in(0,1]$. If $\int_{\partial \Omega} \bar{w} \cdot\left[n \cdot \nabla_{x}\left(x \cdot \nabla_{x} \bar{w}\right)\right] d y>0$, then we have $M(1)-M(1-\delta)=-1$ for $\delta>0$ sufficiently small.

\footnotetext{
${ }^{1}$ We note that a recent result of Wang and W.-M. Ni 22 has shown that the monotonicity of the first positive eigenvalue of the Laplacian for the Neumann boundary condition with respect to the domain is invalid.
} 
The organization of the paper is as follows: in section 3 we discuss properties of the Fredholm Lagrangian Grassmannian $F \Lambda_{\mu}(H)$ and the Maslov index for a continuous curve $\gamma$ in $F \Lambda_{\mu}(H)$, along the lines presented in [14. We have adapted some results from [14 and rephrased them to suit our purposes. In section 4 we give the construction of the Maslov index $\mathbf{M} a s(\{\gamma(t)\}, \mu)$, and finally in section 5 we give the proof of the main results.

\section{Fredholm Lagrangian Grassmannian and monotonicity OF MASLOV INDEX}

In this section we discuss some properties of the Fredholm Lagrangian Grassmannian and the Maslov index. Let $H$ be a real and separable Hilbert space with a symplectic form $\omega$, i.e., a nondegenerate, skew-symmetric bounded bilinear form. Here the nondegeneracy of $\omega$ means that $\omega^{\sharp}: H \rightarrow H^{*}, \omega^{\sharp}(x)(y)=\omega(x, y)$ gives an isomorphism between the Hilbert space $H$ and its dual space $H^{*}$.

For the real separable symplectic Hilbert space $H$ with symplectic form $\omega$, a Lagrangian subspace $L \subset H$ is a maximal closed isotropic subspace of $H$, i.e., a maximal closed subspace on which $\omega(p, q)=0$, for any $p, q \in L$. Let $\Lambda(H)$ denote the set of all the Lagrangian subspaces in $H$, called the Lagrangian Grassmannian of the symplectic Hilbert space $H$. It can be shown that $\Lambda(H)$ is an infinite dimensional differentiable manifold modeled on the Banach space of bounded selfadjoint operators [14]. For two closed subspaces $\mu, \eta$ of $H$, the pair $(\mu, \eta)$ is called a Fredholm pair if $\operatorname{dim}(\mu \cap \eta)<+\infty$, and $\mu+\eta$ is closed and of finite codimension. The Fredholm Lagrangian Grassmannian with respect to $\mu \in \Lambda(H)$ is given by

Definition 3.1. The Fredholm Lagrangian Grassmannian with respect to $\mu \in$ $\Lambda(H)$ is defined as

$$
F \Lambda_{\mu}(H)=\{\eta \in \Lambda(H) \mid(\mu, \eta) \text { is a Fredholm pair }\},
$$

and we call the subset

$$
\mathcal{M}_{\mu}(H)=\left\{\eta \in F \Lambda_{\mu}(H) \mid \eta \cap \mu \neq\{0\}\right\}
$$

the Maslov cycle with respect to $\mu$.

For the symplectic Hilbert space $H$, denote by $G L_{c}(H)$ the Fredholm group of $H$ consisting of linear invertible bounded operators of Id + compact type, and denote by $S p_{c}(H)$ the Fredholm symplectic group consisting of a linear invertible bounded operator $\Psi=I+K$, so that $\omega(x, y)=\omega(\Psi x, \Psi y)$, where $K: H \rightarrow H$ is compact. For a closed subspace $H_{+}$of $H, F_{\text {res }}\left(H_{+}\right)$is the orbit of $H_{+}$under the action of the Fredholm group $G L_{c}(H)$, called the reduced Fredholm Grassmannian based on $H_{+}$. If $H_{+}$is a Lagrangian subspace of $H$, we denote by $F L_{r e s}\left(H_{+}\right)$the orbit of $H_{+}$under the action of the Fredholm symplectic group $S p_{c}(H)$, called the reduced Fredholm Lagrangian Grassmannian based on $H_{+}$.

3.1. Subspaces and Lagrangian subspaces in $H$. In this subsection we list some useful properties about the closed subspaces and Lagrangian subspaces in $H$. First we have

Lemma 3.2. For $\mu, \eta \in \Lambda(H)$, if $\operatorname{dim}(\mu \cap \eta)$ is finite dimensional and $\mu+\eta$ is closed, then $(\mu, \eta)$ is a Fredholm pair. 
Proof. It suffices to show that $\mu+\eta$ is of finite codimension. For any subspace $\xi \in H$, we denote by $\xi^{o}$ the annihilator of $\xi$ with respect to $\omega$ :

$$
\xi^{o}=\{x \in H \quad \mid \omega(x, y)=0, \forall y \in \xi\} .
$$

Thus we have that $\operatorname{dim}\left((\mu+\eta)^{o}\right)=\operatorname{dim}\left(\mu^{o} \cap \eta^{o}\right)=\operatorname{dim}(\mu \cap \eta)<\infty$, where the last equality follows from the fact that $\mu, \eta$ are Lagrangian subspaces. Thus by the nondegeneracy of $\omega$ we have that $\mu+\eta$ is of finite codimension.

Lemma 3.3. Let $\xi \in \Lambda(H)$ and $\eta \in F_{\text {res }}(\xi)$. If $\omega$ vanishes on $\eta$, then $\eta \in \Lambda(H)$, i.e., $\eta$ is a Lagrangian subspace.

Proof. By assumption we have $\eta=\Psi(\xi)$ for some $\Psi \in G L_{c}(H)$. Since $\omega$ vanishes on $\eta,\left.\Psi\right|_{\xi}$ is symplectic; thus we can find $\tilde{\Psi} \in S p_{c}(H)$ so that $\left.\tilde{\Psi}\right|_{\xi}=\left.\Psi\right|_{\xi}$, i.e., $\eta=\tilde{\Psi}(\xi)$. Since $\Lambda(H)$ is invariant under the action of $S p_{c}(H)$, we have that $\eta \in \Lambda(H)$, i.e., $\eta$ is a Lagrangian subspace.

Lemma 3.4. Let $\eta, \mu$ be two closed subspaces of $H$. If $(\eta, \mu)$ is a Fredholm pair, and $\xi \in F_{\text {res }}(\eta)$, then $(\xi, \mu)$ is also a Fredholm pair.

Proof. By assumption $(\eta, \mu)$ is a Fredholm pair; thus we have $\operatorname{dim}(\eta \cap \mu)<+\infty$ and $\operatorname{dim}\left(\eta^{\perp} \cap \mu^{\perp}\right)<+\infty$, where $\eta^{\perp}$ and $\mu^{\perp}$ are the orthogonal complements of $\eta$ and $\mu$ in $H$, respectively. Since $\xi=\Psi(\eta)$ for some $\Psi \in G L_{c}(H), \xi$ is a closed subspace of $H$, and

$$
\operatorname{dim}(\xi \cap \mu)<+\infty, \quad \operatorname{dim}\left(\xi^{\perp} \cap \mu^{\perp}\right)<+\infty,
$$

by a standard functional analysis argument. Thus by definition we have that $(\xi, \mu)$ is also a Fredholm pair.

When the real separable symplectic Hilbert space $H$ has a polarization $H=$ $H_{+} \times H_{+}^{*}$, where $H_{+}^{*}$ is the dual space of $H_{+}$, and the symplectic form

$$
\omega\left(\left(v_{1}, s_{1}\right),\left(v_{2}, s_{2}\right)\right)=\left\langle v_{1}, s_{2}\right\rangle-\left\langle v_{2}, s_{1}\right\rangle, \quad\left(v_{1}, s_{1}\right),\left(v_{2}, s_{2}\right) \in H,
$$

where $\langle$,$\rangle is the pairing between H_{+}$and $H_{+}^{*}$, we have the following.

Lemma 3.5. The subspace $L=\left\{(u, S u) \mid u \in H_{+}\right\}$or $\left\{\left(S^{\prime} v, v\right) \mid v \in H_{+}^{*}\right\}$ is a Lagrangian subspace, where $S: H_{+} \rightarrow H_{+}^{*}$ and $S^{\prime}: H_{+}^{*} \rightarrow H_{+}$are compact and $S^{*}=S, S^{*}=S^{\prime}$.

Proof. Without loss of generality we consider the case of $L=\left\{(u, S u) \mid u \in H_{+}\right\}$. First $L$ is a closed subspace of $H$ by definition. For any $u, u^{\prime} \in H_{+}$, since $S=S^{*}$, we have

$$
\omega\left((u, S u),\left(u^{\prime}, S u^{\prime}\right)\right)=\left\langle u, S u^{\prime}\right\rangle-\left\langle u^{\prime}, S u\right\rangle=0,
$$

which shows that $\omega$ vanishes on $L$. Note that $\omega$ also vanishes on the closed subspace $L_{D}=\left\{(0, v) \mid v \in H_{+}^{*}\right\}$, and $L \oplus L_{D}=H$. If there existed an isotropic closed subspace $L^{\prime} \subset H$ so that $L \subset L^{\prime}, L \neq L^{\prime}$, then there would exist $z \in L^{\prime} \cap L_{D}, z \neq 0$, so that $\omega\left(z, z^{\prime}\right)=0$, for any $z^{\prime} \in L^{\prime}+L_{D}=H$. But this contradicts the nondegeneracy of $\omega$ on $H$ ! Therefore we must have $L=L^{\prime}$, i.e., $L$ is the maximal closed isotropic subspace of $H$, which is equivalent to $L$ being a Lagrangian subspace. 
3.2. Properties of the Maslov index. Let $\gamma:[0,1] \rightarrow F \Lambda_{\mu}(H)$ be a continuous path. There is a well-defined Maslov index $\mathbf{M} a s(\{\gamma(t)\}, \mu)$ which satisfies certain properties [14]. We rephrase and organize the main properties stated in [14] as the following.

Theorem 3.6. For a continuous path $\gamma:[0,1] \rightarrow F \Lambda_{\mu}(H)$, the Maslov index $\mathbf{M} a s(\{\gamma(t)\}, \mu)$ satisfies the following properties:

- 1) If $\gamma(t) \notin \mathcal{M}_{\mu}(H)$, for each $t \in[0,1]$, then $\mathbf{M} a s(\{\gamma(t)\}, \mu)=0$.

- 2) Additivity under the catenation of the paths.

- 3) Modulo sign and addition constants, it is only a homotopy invariant of curves in $F \Lambda_{\mu}(H)$ with fixed endpoints and distinguishes the homotopy classes.

- 4) Let $\xi \in F L_{r e s}(\mu)$. Then $\mathbf{M} a s(\{\gamma(t)\}, \mu)-\mathbf{M} a s(\{\gamma(t)\}, \xi)$ depends only on the end points.

Proof. Property (1) follows from the definition of the Maslov index $\mathbf{M} a s(\{\gamma(t)\}, \mu)$, while properties (2) and (3) follow from Theorem 1.54 and Theorem 2.6 of [14], and property (4) is a restatement of Proposition 2.11 of [14.

In the case of $\gamma:[0,1] \rightarrow F \lambda_{\mu}(H)$ being a $C^{1}$ path, following [14, we call $t^{*} \in[0,1]$ a crossing for the family $\{\gamma(t)\}$, if $\gamma\left(t^{*}\right) \cap \mu \neq\{0\}$. To introduce the crossing form at $t=t^{*}$, let $\nu$ be a Lagrangian subspace which is transversal to $\gamma\left(t^{*}\right)$. Then $\gamma(t)$ is transversal to $\nu$ for sufficiently small $\left|t-t^{*}\right| \ll 1, t \in[0,1]$. Therefore there exists a differentiable family of bounded operators $\left\{\phi_{t}\right\}_{\left|t-t^{*}\right| \ll 1, t \in[0,1]}, \phi_{t}$ : $\gamma\left(t^{*}\right) \rightarrow \nu$, so that $\gamma(t)=$ graph of $\phi_{t}$, for $\left|t-t^{*}\right| \ll 1, t \in[0,1]$. The bilinear form $\left.Q_{\mathcal{M}}(x, y) \stackrel{\text { def }}{=} \frac{d}{d t} \omega\left(x, \phi_{t}(y)\right)\right|_{t=t^{*}}$, for $x, y \in \gamma\left(t^{*}\right) \cap \mu$ is called the crossing form at $t=t^{*}$. It can be shown that the crossing form $Q_{\mathcal{M}}(x, y)$ does not depend on the choice of $\nu$ [14. This notion will be of central importance for us, since it offers us a direct way of calculating the Maslov index locally. By rephrasing Remark 2.24, Proposition 2.27 and Proposition 2.29 of [14] we have that

Proposition 3.7. Let $\gamma:[0,1] \rightarrow F \Lambda_{\mu}(H)$ be a $C^{1}$ path, and let $0 \leq t^{*} \leq 1$ be a crossing of the curve. If $\left.Q_{\mathcal{M}}\right|_{\gamma\left(t^{*}\right) \cap \mu}$ is nondegenerate, then there exists a positive $\delta>0$ such that 1) for $t^{*} \in(0,1)$,

$$
\mathbf{M} a s\left(\{\gamma(t)\}_{\left|t-t^{*}\right| \leq \delta}, \mu\right)=\operatorname{sign} Q_{\mathcal{M}},
$$

2) for $t^{*}=0$ or 1 ,

$$
\operatorname{M} a s\left(\{\gamma(t)\}_{0 \leq t \leq \delta}, \mu\right)=-q, \quad \mathbf{M} a s\left(\{\gamma(t)\}_{1-\delta \leq t \leq 1}, \mu\right)=p^{\prime},
$$

where sign $Q_{\mathcal{M}}$ is the signature of $Q_{\mathcal{M}}$ on the finite dimensional subspace $\gamma\left(t^{*}\right) \cap \mu$, and the positive/negative index of inertia of $Q_{\mathcal{M}}$ at $t^{*}=0$ is $(p, q)$ and at $t^{*}=1$ is $\left(p^{\prime}, q^{\prime}\right)$.

The crossing $t^{*} \in[0,1]$ will be called a regular crossing if $\left.Q_{\mathcal{M}}\right|_{\gamma\left(t^{*}\right) \cap \mu}$ is nondegenerate. Any regular crossing point $t^{*}$ will then be an isolated point in $[0,1]$. It is called a positive (negative) crossing if $\left.Q_{\mathcal{M}}\right|_{\gamma\left(t^{*}\right) \cap \mu}$ is positive (negative) definite. The curve $\gamma$ will be called a positive (negative) curve if each crossing is a positive (negative) crossing. A positive (negative) curve $\gamma:[0,1] \rightarrow F \Lambda_{\mu}(H)$ will thus have only finitely many crossings.

One distinct characteristic of positive (negative) curves is the monotonicity of the Maslov index with respect to $t$, which is exhibited by the following. 
Proposition 3.8. Let $\gamma:[0,1] \rightarrow F \Lambda_{\mu}(H)$ be a $C^{1}$ positive curve. Then we have

$$
\operatorname{Mas}(\{\gamma(t)\}, \mu)=\sum_{0<t_{i} \leq 1} \operatorname{dim}\left(\gamma\left(t_{i}\right) \cap \mu\right),
$$

where $t_{i} \in[0,1]$ is the position of positive crossings.

Proof. Since $\gamma$ is a positive curve, it will necessarily have only finitely many crossings $t_{1}, t_{2}, \ldots, t_{k} \in[0,1]$, with corresponding $\delta_{i}, i=1,2, \ldots, k$ guaranteed by Proposition 3.7. Let $\delta=\frac{1}{2} \min _{1 \leq i \leq k}\left\{\delta_{i}\right\}$. We split the interval $[0,1]$ into closed subintervals $I_{j}, 1 \leq j \leq s$, some $s \geq 3$ of length no more than $\delta$, such that for each $i \in\{1, \ldots, k\}$, if $t_{i} \neq 0$ nor 1 , then $t_{i}$ lies in the interior of some $I_{j}, 2 \leq j \leq s-1$, and $I_{j}$ contains only that crossing point; if $t_{i}=0$, then $t_{i} \in I_{1}$; if $t_{i}=1$, then $t_{i} \in I_{s}$.

For $2 \leq j \leq s-1$, if $I_{j}$ contains no crossing point, then $\mathbf{M} a s\left(\{\gamma(t)\}_{t \in I_{j}}, \mu\right)=0$, due to property (1) of the Maslov index in Theorem 3.6. If $I_{j}$ contains one crossing point $t_{i}$, then $\mathbf{M} a s\left(\{\gamma(t)\}_{t \in I_{j}}, \mu\right)=\left.\operatorname{sign} Q_{\mathcal{M}}\right|_{\gamma\left(t_{i}\right) \cap \mu}=\operatorname{dim}\left(\gamma\left(t_{i}\right) \cap \mu\right)$, by Proposition 3.7 and that sign $\left.Q_{\mathcal{M}}\right|_{\gamma\left(t_{i}\right) \cap \mu}=\operatorname{dim}\left(\gamma\left(t_{i}\right) \cap \mu\right)$, since $\gamma$ is a positive curve. Equation (3.1) then follows from property (2) of the Maslov index of Theorem 3.6 and the calculation of the Maslov index for crossing $t=0$ or 1 as in Proposition 3.7 .

Corollary 3.9. Let $\gamma:[0,1] \rightarrow F \Lambda_{\mu}(H)$ be a $C^{1}$ negative curve. Then we have

$$
\mathbf{M} a s(\{\gamma(t)\}, \mu)=-\sum_{0 \leq t_{i}<1} \operatorname{dim}\left(\gamma\left(t_{i}\right) \cap \mu\right),
$$

where $t_{i} \in[0,1]$ is the position of negative crossings.

If $\left.Q_{\mathcal{M}}\right|_{\gamma\left(t^{*}\right) \cap \mu} \equiv 0$ on $\gamma\left(t^{*}\right) \cap \mu$, and the curve $\gamma$ is of $C^{2}$, then we can consider the secondary crossing form

$$
\left.Q_{\mathcal{M}}^{(2)}(x, y) \stackrel{\text { def }}{=} \frac{d^{2}}{d t^{2}} \omega\left(x, \phi_{t}(y)\right)\right|_{t=t^{*}}
$$

for $x, y \in \gamma\left(t^{*}\right) \cap \mu$. Similar to the proof of Proposition 3.7 we have that

Proposition 3.10. Let $\gamma:[0,1] \rightarrow F \Lambda_{\mu}(H)$ be a $C^{2}$ path, and let $0 \leq t^{*} \leq 1$

be a crossing of the curve. If $\left.Q_{\mathcal{M}}\right|_{\gamma\left(t^{*}\right) \cap \mu} \equiv 0$ on $\gamma\left(t^{*}\right) \cap \mu$, and $\left.Q_{\mathcal{M}}^{(2)}\right|_{\gamma\left(t^{*}\right) \cap \mu}$ is nondegenerate, then there would exist a positive $\delta>0$ such that 1) for $t^{*} \in(0,1)$,

$$
\mathbf{M} a s\left(\{\gamma(t)\}_{t^{*} \leq t \leq t^{*}+\delta}, \mu\right)=-\mathbf{M} a s\left(\{\gamma(t)\}_{t^{*}-\delta \leq t \leq t^{*}}, \mu\right)=-q,
$$

2) for $t^{*}=0$ or 1 ,

$$
\mathbf{M} a s\left(\{\gamma(t)\}_{0 \leq t \leq \delta}, \mu\right)=-q, \quad \mathbf{M} a s\left(\{\gamma(t)\}_{1-\delta \leq t \leq 1}, \mu\right)=q^{\prime},
$$

where the positive/negative index of inertia of $Q_{\mathcal{M}}^{(2)}$ at $t^{*} \in[0,1)$ is $(p, q)$ and at $t^{*}=1$ is $\left(p^{\prime}, q^{\prime}\right)$.

\section{Domain Shrinking AND CONSTRUCTION Of PATH in Fredholm LAGRANGIAN GRASSMANNIAN}

In this section we fix the real separable symplectic Hilbert space as $H=H^{\frac{1}{2}}(\partial \Omega)$ $\times H^{-\frac{1}{2}}(\partial \Omega)$, and the symplectic form

$$
\omega\left(\left(v_{1}, s_{1}\right),\left(v_{2}, s_{2}\right)\right)=\left\langle v_{1}, s_{2}\right\rangle-\left\langle v_{2}, s_{1}\right\rangle, \quad\left(v_{1}, s_{1}\right),\left(v_{2}, s_{2}\right) \in H,
$$

where $\langle$,$\rangle is the pairing between H^{\frac{1}{2}}(\partial \Omega)$ and $H^{-\frac{1}{2}}(\partial \Omega)$. 
Let the hypothesis (Ha) be satisfied, and assume that $\bar{u} \in C^{2}(\bar{\Omega})$. We now detail the domain-shrinking method to construct a $C^{2}$ path in the Fredholm Lagrangian Grassmannian $F \Lambda_{\mu}(H)$, where $\mu=\operatorname{ker} P$. Recall that the shrunken domain $\Omega_{t}=$ $\{x \in \Omega: x=s y, 0 \leq s<t, y \in \partial \Omega\}$, and $H_{t}=H^{\frac{1}{2}}\left(\partial \Omega_{t}\right) \times H^{-\frac{1}{2}}\left(\partial \Omega_{t}\right), 0<t \leq 1$. (Thus $H=H_{1}$.) The symplectic form for $H_{t}$ has the same form as that for $H$.

We consider the following:

$$
-\triangle_{x} w+t^{2} f(t x) w=t^{2} \lambda w, \quad x \in \Omega,
$$

where $t \in[0,1], \lambda \in R$. For $t \in(0,1]$, it is obtained by scaling equation (2.3) from $\Omega_{t}$ to $\Omega$. Denoting by $\tilde{K}_{\lambda, t}$ the set of all $H^{1}$ weak solutions for (4.1), for each $t \in(0,1]$, the trace map $\tilde{T}_{t}: H^{1}(\Omega) \rightarrow H$ is given by $\tilde{T}_{t}(u)=\left(\left.u\right|_{\partial \Omega},\left.\frac{1}{t} \cdot \frac{\partial u}{\partial n}\right|_{\partial \Omega}\right)$, for $u \in H^{1}(\Omega)$. First we have the following.

Proposition 4.1. $\tilde{T}_{t}\left(\tilde{K}_{\lambda, t}\right) \in F \Lambda_{\mu}(H)$ for each $t \in(0,1], \lambda \in R$.

Proof. We follow the lines of the proof for the transversality lemma of [8]. Fix $\lambda \in$ $R, t \in(0,1]$. Let $\tilde{H} \stackrel{\text { def }}{=} H^{1}(\Omega)$, and the polarization of $\tilde{H}$ be given by $H_{0}^{1}(\Omega) \oplus \tilde{L}_{c}$, where $H_{0}^{1}(\Omega)$ consists of functions in $H^{1}(\Omega)$ with zero trace on $\partial \Omega$, and $\tilde{L}_{c} \stackrel{\text { def }}{=}\{u \in$ $\left.H^{1}(\Omega), \mid-\triangle u=0\right\}$, i.e., the set of harmonic functions in $\Omega$. For each $z \in H^{1}(\Omega)$, we have $z=z_{1}+z_{2}$, where $z_{1} \in H_{0}^{1}(\Omega), z_{2} \in \tilde{L}_{c}$. This induces two projections $\pi_{1}, \pi_{2}: \tilde{H} \rightarrow \tilde{H}, \pi_{1}(z)=z_{1}, \pi_{2}(z)=z_{2}$. We denote by $L_{c, t}$ the image of $\tilde{L}_{c}$ under $\tilde{T}_{t}$, i.e., $L_{c, t}=\tilde{T}_{t}\left(\tilde{L}_{c}\right)$. Note that $L_{c, t}$ is a Lagrangian subspace of $H$.

For $w \in \tilde{H}$, Equation (4.1) can now be written as

$$
w-F(\lambda, t)(w)=\pi_{2} w,
$$

where $F(\lambda, t): \tilde{H} \rightarrow H_{0}^{1}(\Omega), F(\lambda, t)(w) \stackrel{\text { def }}{=}(-\triangle)^{-1}\left[-t^{2} f(t x) w+t^{2} \lambda w\right]$ is compact. Define

$$
G(\lambda, t): \tilde{H} \rightarrow \tilde{H}, G(\lambda, t) w \stackrel{\text { def }}{=} w-F(\lambda, t) w .
$$

Then arguing exactly as given in [8], we have $\tilde{K}_{\lambda, t}=G^{-1}(\lambda, t)\left(\tilde{L}_{c}\right)$ and that

$$
G(\lambda, t)(\tilde{H})+\pi_{2}(\tilde{H})=G(\lambda, t)(\tilde{H})+\tilde{L}_{c}=\tilde{H} .
$$

Since $G$ is of Id + compact type, using Lemma 6 of [7] we know that $\tilde{K}_{\lambda, t}=$ $G^{-1}(\lambda, t)\left(\tilde{L}_{c}\right) \in F_{\text {res }}\left(\tilde{L}_{c}\right)$, thus $\tilde{T}_{t}\left(\tilde{K}_{\lambda, t}\right) \in F_{\text {res }}\left(L_{c, t}\right)$, by noticing that $\tilde{T}_{t}\left(\tilde{L}_{c}\right)=L_{c, t}$ and the map $\tilde{T}_{t}: \tilde{K}_{\lambda, t} \rightarrow \tilde{T}_{t}\left(\tilde{K}_{\lambda, t}\right)$ is an isomorphism, following Lemma 6 of [8].

To show that $\left(L_{c, t}, \mu\right)$ is a Fredholm pair, note that $L_{c, t}$ is the image of all harmonic functions in $\Omega$ under the trace map $\tilde{T}_{t}$; thus we have $L_{c, t}=\left\{\left(u, \frac{1}{t} \mathbf{N} u\right) \mid u \in\right.$ $\left.H^{\frac{1}{2}}(\partial \Omega)\right\}$, where $\mathbf{N}: H^{\frac{1}{2}}(\partial \Omega) \rightarrow H^{-\frac{1}{2}}(\partial \Omega)$ is the standard Dirichlet-to-Neumann operator [20]. Letting $L_{D} \stackrel{\text { def }}{=}\left\{(0, v) \mid v \in H^{-\frac{1}{2}}(\partial \Omega)\right\}, L_{N} \stackrel{\text { def }}{=}\left\{(u, 0) \mid u \in H^{\frac{1}{2}}(\partial \Omega)\right\}$, we have that $\left(L_{c, t}, L_{D}\right),\left(L_{c, t}, L_{N}\right)$ are both Fredholm pairs since $L_{c, t} \oplus L_{D}=H$, $L_{c, t} \cap L_{N}=\left\{\left(\left.c \cdot 1\right|_{\partial \Omega}, 0\right) \mid c \in R\right\}$ is one-dimensional, $L_{c, t}+L_{N}$ is closed and of codimension one due to the fact that $\mathbf{N}: H^{\frac{1}{2}}(\partial \Omega) \rightarrow H^{-\frac{1}{2}}(\partial \Omega)$ is Fredholm and of index 0 20. By assumption (Ha), $\mu$ is either Dirichlet-based or Neumann-based, which means that $\mu \in F_{\text {res }}\left(L_{D}\right)$ or $\mu \in F_{\text {res }}\left(L_{N}\right)$; thus by Lemma 3.4 of section 3 we have that $\left(L_{c, t}, \mu\right)$ is a Fredholm pair. From the paragraph above we know that $\tilde{T}_{t}\left(\tilde{K}_{\lambda, t}\right) \in F_{r e s}\left(L_{c, t}\right)$; using Lemma 3.4 once more, we have that $\left(\tilde{T}_{t}\left(\tilde{K}_{\lambda, t}\right), \mu\right)$ is a Fredholm pair.

Thus the proof will be complete if we show that $\tilde{T}_{t}\left(\tilde{K}_{\lambda, t}\right)$ is a Lagrangian subspace in $H$. But since it is easy to check that $\omega$ vanishes on $\tilde{T}_{t}\left(\tilde{K}_{\lambda, t}\right)$, and we have 
$\tilde{T}_{t}\left(\tilde{K}_{\lambda, t}\right) \in F_{r e s}\left(L_{c, t}\right)$, therefore by Lemma 3.3 of section 3 we get that $\tilde{T}_{t}\left(\tilde{K}_{\lambda, t}\right)$ is a Lagrangian subspace of $H$.

Remark 4.2. Notice that $G \in C^{2}\left(R \times[0,1] ; G L_{c}(\tilde{H}, \tilde{H})\right)$ if $\bar{u} \in C^{2}(\bar{\Omega})$.

Denoting $W^{u}(\lambda, t): R \times(0,1] \rightarrow F \Lambda_{\mu}(H), W^{u}(\lambda, t) \stackrel{\text { def }}{=} \tilde{T}_{t}\left(\tilde{K}_{\lambda, t}\right)$, then we have

Proposition 4.3. $W^{u} \in C^{2}\left(R \times(0,1] ; F \Lambda_{\mu}(H)\right)$.

Proof. Since $\tilde{K}_{\lambda, t}=G^{-1}(\lambda, t)\left(\tilde{L}_{c}\right)$, we have that $W^{u}(\lambda, t)=\tilde{T}_{t}\left(G^{-1}(\lambda, t)\left(\tilde{L}_{c}\right)\right)$, whereas by Remark 4.2, $G \in C^{2}\left(R \times[0,1] ; G L_{c}(\tilde{H}, \tilde{H})\right)$; thus by Equation (4.3) and Remark 7 of [7] we get that $W^{u} \in C^{2}\left(R \times(0,1] ; F \Lambda_{\mu}(H)\right)$.

Lemma 4.4. For each $t \in(0,1]$, the scaling map $\Phi_{t}: \partial \Omega \rightarrow \partial \Omega_{t}$ given by $\Phi_{t}(y)=$ ty induces a symplectic map $\tilde{\Phi}_{t}:\left(H_{t}, \omega\right) \rightarrow\left(H, t^{m-1} \omega\right)$ by defining

$$
\tilde{\Phi}_{t}(u(z), v(z))=(\tilde{u}(y), \tilde{v}(y)),
$$

where $z=t y \in \partial \Omega_{t}, \tilde{u}(y)=u(t y), \tilde{v}(y)=v(t y)$.

Proof. First notice that $\tilde{\Phi}_{t}$ is an isomorphism between $H_{t}=H^{\frac{1}{2}}\left(\partial \Omega_{t}\right) \times H^{-\frac{1}{2}}\left(\partial \Omega_{t}\right)$ and $H=H^{\frac{1}{2}}(\partial \Omega) \times H^{-\frac{1}{2}}(\partial \Omega)$, due to the fact that $C^{\infty}\left(\partial \Omega_{t}\right)$ for $t \in(0,1]$ is dense in $H^{\frac{1}{2}}\left(\partial \Omega_{t}\right)$. We then only need to check that

$$
t^{m-1} \omega\left(\tilde{\Phi}_{t}\left(u_{1}, v_{1}\right), \tilde{\Phi}_{t}\left(u_{2}, v_{2}\right)\right)=\omega\left(\left(u_{1}, v_{1}\right),\left(u_{2}, v_{2}\right)\right)
$$

holds for any $\left(u_{1}, v_{1}\right),\left(u_{2}, v_{2}\right) \in H_{t}$, i.e.,

$$
t^{m-1} \int_{\partial \Omega}\left(v_{2}(t y) \cdot u_{1}(t y)-v_{1}(t y) \cdot u_{2}(t y)\right) d y=\int_{\partial \Omega_{t}}\left(v_{2}(z) \cdot u_{1}(z)-v_{1}(z) \cdot u_{2}(z)\right) d z
$$

which holds by letting $z=t y$ and using the chain rule for integration.

Remark 4.5. It is interesting to notice that, although $\tilde{\Phi}_{t}:\left(H_{t}, \omega\right) \rightarrow(H, \omega)$ is not symplectic, $\tilde{\Phi}_{t}$ still maps each Lagrangian subspace of $\left(H_{t}, \omega\right)$ into a Lagrangian subspace of $(H, \omega)$, and vice versa, on account of Equation (4.6) above.

Recall that $K_{\lambda, t}$ is the set of weak solutions of (2.3) in $H^{1}\left(\Omega_{t}\right), t \in(0,1]$. For each $t \in(0,1]$, we use the following trace map $T_{t}: K_{\lambda, t} \rightarrow H_{t}$ :

$$
T_{t}(w)=\left(\left.w\right|_{\partial \Omega_{t}},\left.\frac{\partial w}{\partial n}\right|_{\partial \Omega_{t}}\right), \quad w \in K_{\lambda, t} .
$$

We have the following.

Lemma 4.6. For each $\lambda \in R, t \in(0,1], \tilde{\Phi}_{t}\left(T_{t}\left(K_{\lambda, t}\right)\right)=W^{u}(\lambda, t)$.

Proof. Fix $\lambda \in R, t \in(0,1]$. The statement follows from the observation that $w(\cdot)$ is a weak solution of (4.1) in $H^{1}(\Omega)$ if and only if $w(\dot{\bar{t}})$ is a weak solution of (2.3) in $H^{1}\left(\Omega_{t}\right)$, and for $x \in \partial \Omega, y=t x \in \partial \Omega_{t}$, we have

$$
\mathbf{n}(y) \cdot \nabla_{y} w\left(\frac{y}{t}\right)=\frac{1}{t} \cdot \mathbf{n}(x) \cdot \nabla_{x} w(x) .
$$

Lemma 4.7. For each $t \in(0,1], W^{u}(\cdot, t): \lambda \in(-\infty, 0] \rightarrow F \Lambda_{\mu}(H)$ is a negative curve in $F \Lambda_{\mu}(H)$. 
Proof. Fix $t \in(0,1]$. Let $\lambda_{0} \in(-\infty, 0]$ be a crossing of $W^{u}((-\infty, 0], t)$ in $F \Lambda_{\mu}(H)$, i.e., $W^{u}\left(\lambda_{0}, t\right) \cap \mu \neq\{0\}$. It suffices to show that $\lambda_{0}$ is a negative crossing. Now let $\nu$ be a Lagrangian subspace which is transversal to $W^{u}\left(\lambda_{0}, t\right)$. Then $W^{u}(\cdot, t)$ is transversal to $\nu$ for sufficiently small $\left|\lambda-\lambda_{0}\right| \ll 1$. Therefore there exists a $C^{1}$ differentiable family of bounded operators $\left\{\phi_{\lambda}\right\}_{\left|\lambda-\lambda_{0}\right| \ll 1}, \phi_{\lambda}: W^{u}\left(\lambda_{0}, t\right) \rightarrow \nu$, so that $W^{u}(\lambda, t)=$ graph of $\phi_{\lambda}$, for $\left|\lambda-\lambda_{0}\right| \ll 1, \lambda \in(-\infty, 0]$.

Fix $q \in W^{u}\left(\lambda_{0}, t\right) \cap \mu, q \neq 0$. We consider the curve

$$
X(\lambda)=q+\phi_{\lambda}(q) \in W^{u}(\lambda, t),\left|\lambda-\lambda_{0}\right| \ll 1 .
$$

By definition of $W^{u}(\lambda, t)$, there exists a $C^{1}$ curve of solutions $w(\lambda)$ for

$$
-\triangle w+t^{2} f(t x) w=t^{2} \lambda w
$$

such that the trace map $\left(\left.w(\lambda)\right|_{\partial \Omega},\left.\frac{1}{t} \cdot \frac{\partial w(\lambda)}{\partial n}\right|_{\partial \Omega}\right) \in H$ is exactly $X(\lambda)$. We claim the following.

Claim 4.8. $\omega\left(X\left(\lambda_{0}\right), \frac{\partial X}{\partial \lambda}\left(\lambda_{0}\right)\right)<0$.

Proof of Claim 4.8. We have

$$
\omega\left(X, \frac{\partial X}{\partial \lambda}\right)=\frac{1}{t} \cdot \int_{\partial \Omega} w \frac{\partial^{2} w}{\partial \lambda \partial n}-\frac{\partial w}{\partial \lambda} \cdot \frac{\partial w}{\partial n} d y
$$

whereas, from (4.9) we have

$$
\begin{array}{r}
-\triangle \frac{\partial w}{\partial \lambda}-t^{2} w+\left(t^{2} f(t x)-t^{2} \lambda\right) \frac{\partial w}{\partial \lambda}=0, \\
\frac{\partial X}{\partial \lambda}=\left(\left.\frac{\partial w}{\partial \lambda}\right|_{\partial \Omega},\left.\frac{1}{t} \cdot \frac{\partial^{2} w}{\partial \lambda \partial n}\right|_{\partial \Omega}\right) \in \nu .
\end{array}
$$

By multiplying (4.11) with $w$ and integrating over $\Omega$, we have

$$
\begin{aligned}
\int_{\partial \Omega} w \frac{\partial^{2} w}{\partial \lambda \partial n} d y & =\iint_{\Omega} \nabla w \cdot \nabla\left(\frac{\partial w}{\partial \lambda}\right)+\frac{\partial w}{\partial \lambda} \cdot\left(t^{2} f(t x)-t^{2} \lambda\right) w-t^{2} w^{2} d x \\
& =\int_{\partial \Omega} \frac{\partial w}{\partial n} \cdot \frac{\partial w}{\partial \lambda} d y+\iint_{\Omega} \frac{\partial w}{\partial \lambda} \cdot\left(-\triangle+t^{2} f(t x)-t^{2} \lambda\right) w-t^{2} w^{2} d x
\end{aligned}
$$

Combining (4.10) and (4.13) we have

$$
\omega\left(X, \frac{\partial X}{\partial \lambda}\right)=\frac{1}{t} \cdot \iint_{\Omega} \frac{\partial w}{\partial \lambda} \cdot\left(-\triangle+t^{2} f(t x)-t^{2} \lambda\right) w-t^{2} w^{2} d x=-t \iint_{\Omega} w^{2} d x
$$

Thus at $\lambda=\lambda_{0}$ we have

$$
\omega\left(X\left(\lambda_{0}\right), \frac{\partial X}{\partial \lambda}\left(\lambda_{0}\right)\right)=-t \iint_{\Omega} w^{2}\left(\lambda_{0}\right) d x<0,
$$

which finishes the proof of Claim 4.8.

Since for each $q \in W^{u}\left(\lambda_{0}, t\right) \cap \mu, q \neq 0$, we have

$$
\begin{aligned}
\left.Q_{\mathcal{M}}(q, q) \stackrel{\text { def }}{=} \frac{d}{d \lambda}\right|_{\lambda=\lambda_{0}} \omega\left(q, \phi_{\lambda}(q)\right) & =\left.\frac{d}{d \lambda}\right|_{\lambda=\lambda_{0}} \omega\left(q, q+\phi_{\lambda}(q)\right) \\
& =\omega\left(X\left(\lambda_{0}\right), \frac{\partial X}{\partial \lambda}\left(\lambda_{0}\right)\right)<0
\end{aligned}
$$

due to Claim 4.8, thus by definition, $\lambda=\lambda_{0}$ is a negative crossing for the curve $W^{u}(\cdot, t)$. The proof of Lemma 4.7 is complete. 
Lemma 4.9. For any $t_{0} \in(0,1]$, there exists $\lambda\left(t_{0}\right)<0$ such that $W^{u}(\lambda, t) \notin$ $\mathcal{M}_{\mu}(H)$ for $\lambda \leq \lambda\left(t_{0}\right), t \in\left[t_{0}, 1\right]$.

Proof. We give the proof that ker $P$ is Neumann based, i.e., $\operatorname{ker} P=\{(u, S u) \mid u \in$ $\left.H^{\frac{1}{2}}(\partial \Omega)\right\}$, where $S: H^{\frac{1}{2}}(\partial \Omega) \rightarrow H^{-\frac{1}{2}}(\partial \Omega)$ is compact and $S^{*}=S$. The proof for the case of ker $P$ being Dirichlet based is similar.

Fix $t_{0} \in(0,1]$. Denote $A_{\lambda, t}=-\triangle+t^{2}(f(t x)-\lambda)$ and $H_{P, t} \stackrel{\text { def }}{=}\left\{u \in H^{2}(\Omega) \mid\left(\left.u\right|_{\partial \Omega}\right.\right.$, $\left.\left.\left.\frac{1}{t} \cdot \frac{\partial u}{\partial n}\right|_{\partial \Omega}\right) \in \operatorname{ker} P\right\}$, for $\lambda \leq 0, t \in\left[t_{0}, 1\right]$. Since $S=S^{*}: H^{\frac{1}{2}}(\partial \Omega) \rightarrow H^{-\frac{1}{2}}(\partial \Omega)$ is compact, we have a decomposition $H^{\frac{1}{2}}(\partial \Omega)=H_{1} \oplus H_{2}$ such that $\operatorname{dim}\left(H_{1}\right)<+\infty$, and $\langle u, S u\rangle \leq \frac{1}{2 C^{2}}\|u\|_{H^{\frac{1}{2}}(\partial \Omega)}^{2}$ for any $u \in H_{2}$, where $C>0$ is the constant that satisfies $\left\|\left.u\right|_{\partial \Omega}\right\|_{H^{\frac{1}{2}(\partial \Omega)}} \leq C\|u\|_{H^{1}(\Omega)}$.

Letting $\lambda_{1}\left(t_{0}\right)=-\|f(x)\|_{L^{\infty}(\Omega)}-\frac{1}{t_{0}^{2}}$, we claim the following.

Claim 4.10. For $t \in\left[t_{0}, 1\right], \lambda \leq \lambda_{1}\left(t_{0}\right), A_{\lambda, t}$ is a strictly positive selfadjoint operator when restricted to $\left\{u \in H^{2}(\Omega)\left|\left(\left.u\right|_{\partial \Omega},\left.\frac{1}{t} \cdot \frac{\partial u}{\partial n}\right|_{\partial \Omega}\right) \in \operatorname{ker} P, u\right|_{\partial \Omega} \in H_{2}\right\}$.

Proof of Claim 4.10. First $A_{\lambda, t}$ when restricted to

$$
H_{P, t}=\left\{u \in H^{2}(\Omega) \mid\left(\left.u\right|_{\partial \Omega},\left.\frac{1}{t} \cdot \frac{\partial u}{\partial n}\right|_{\partial \Omega}\right) \in \operatorname{ker} P\right\}
$$

is a selfadjoint operator by direct calculation. For $t \in\left[t_{0}, 1\right], \lambda \leq \lambda_{1}\left(t_{0}\right)$, any $u \in\left\{u \in H^{2}(\Omega)\left|\left(\left.u\right|_{\partial \Omega},\left.\frac{1}{t} \cdot \frac{\partial u}{\partial n}\right|_{\partial \Omega}\right) \in \operatorname{ker} P, u\right|_{\partial \Omega} \in H_{2}\right\}$, we have

$$
\begin{array}{r}
\left(A_{\lambda, t} u, u\right)_{L^{2}(\Omega)}=-\int_{\partial \Omega} \frac{\partial u}{\partial n} u d y+\iint_{\Omega}|\nabla u|^{2}+t^{2}(f(t x)-\lambda) u^{2} d x \\
=-\int_{\partial \Omega} t\langle u, K u\rangle d y+\iint_{\Omega}|\nabla u|^{2}+t^{2}(f(t x)-\lambda) u^{2} d x \\
\quad \geq\|u\|_{H^{1}(\Omega)}^{2}-\frac{1}{2 C^{2}}\left\|\left.u\right|_{\partial \Omega}\right\|_{H^{\frac{1}{2}(\partial \Omega)}}^{2} \geq \frac{1}{2}\|u\|_{H^{1}(\Omega)}^{2}
\end{array}
$$

thus $A_{\lambda, t}$ is positive when restricted to $\left\{u \in H^{2}(\Omega)\left|\left(\left.u\right|_{\partial \Omega},\left.\frac{1}{t} \cdot \frac{\partial u}{\partial n}\right|_{\partial \Omega}\right) \in \operatorname{ker} P, u\right|_{\partial \Omega} \in\right.$ $\left.H_{2}\right\}$. The proof of Claim 4.10 is complete.

Let the subspace $U_{\lambda, t} \subset H_{P, t}, \lambda \leq \lambda_{1}\left(t_{0}\right), t \in\left[t_{0}, 1\right]$ be such that

$$
U_{\lambda, t} \oplus\left\{u \in H^{2}(\Omega)\left|\left(\left.u\right|_{\partial \Omega},\left.\frac{1}{t} \cdot \frac{\partial u}{\partial n}\right|_{\partial \Omega}\right) \in \operatorname{ker} P, u\right|_{\partial \Omega} \in H_{2}\right\}=H_{P, t},
$$

and $\left(u, A_{\lambda, t} v\right)_{L^{2}(\Omega)}=0$ for $u \in U_{\lambda, t}, v \in\left\{u \in H^{2}(\Omega)\left|\left(\left.u\right|_{\partial \Omega},\left.\frac{1}{t} \cdot \frac{\partial u}{\partial n}\right|_{\partial \Omega}\right) \in \operatorname{ker} P, u\right|_{\partial \Omega}\right.$ $\left.\in H_{2}\right\}$. We have $\operatorname{dim}\left(U_{\lambda, t}\right)=\operatorname{dim}\left(H_{1}\right)<+\infty$; therefore for each $t \in\left[t_{0}, 1\right]$, there exists $\lambda_{2}(t)<\lambda_{1}\left(t_{0}\right)<0$, so that $A_{\lambda, t}$ restricted to $U_{\lambda, t}$ is positive definite whenever $\lambda \leq \lambda_{2}(t), t \in\left[t_{0}, 1\right]$. Without loss we can choose $\lambda_{2}(t)$ to be a bounded function on $t \in\left[t_{0}, 1\right]$, due to the compactness of the interval $\left[t_{0}, 1\right]$.

Now let $\lambda\left(t_{0}\right)=\inf _{t \in\left[t_{0}, 1\right]} \lambda_{2}(t)$. Then for $\lambda \leq \lambda\left(t_{0}\right), t \in\left[t_{0}, 1\right]$ and any $u \in H_{P, t}$, we have $u=u_{1}+u_{2}$, where $u_{1} \in U_{\lambda, t}, u_{2} \in\left\{u \in H^{2}(\Omega) \mid\left(\left.u\right|_{\partial \Omega},\left.\frac{1}{t} \cdot \frac{\partial u}{\partial n}\right|_{\partial \Omega}\right) \in\right.$ ker $\left.P,\left.u\right|_{\partial \Omega} \in H_{2}\right\}$, and

$$
\left(A_{\lambda, t} u, u\right)_{L^{2}(\Omega)}=\left(A_{\lambda, t} u_{1}, u_{1}\right)_{L^{2}(\Omega)}+\left(A_{\lambda, t} u_{2}, u_{2}\right)_{L^{2}(\Omega)} \geq C^{\prime}\left\|\left.u\right|_{\partial \Omega}\right\|_{H^{\frac{1}{2}}(\partial \Omega)}^{2},
$$


for some $C^{\prime}>0$. Therefore we have no nontrivial solution $w$ for the following equation:

$$
\begin{gathered}
-\triangle w+t^{2} f(t x) w=t^{2} \lambda w, \\
\left(\left.w\right|_{\partial \Omega},\left.\frac{1}{t} \cdot \frac{\partial w}{\partial n}\right|_{\partial \Omega}\right) \in \operatorname{ker} P,
\end{gathered}
$$

which is equivalent to $W^{u}(\lambda, t) \notin \mathcal{M}_{\mu}(H)$ for $\lambda \leq \lambda\left(t_{0}\right), t \in\left[t_{0}, 1\right]$. The proof is complete.

Remark 4.11. It follows from the proof of Lemma 4.9 that for any $t_{0} \in(0,1], A_{\lambda, t}=$ $-\triangle+t^{2}(f(t x)-\lambda)$ is positive definite on $H_{P, t} \stackrel{\text { def }}{=}\left\{u \in H^{2}(\Omega) \mid\left(\left.u\right|_{\partial \Omega},\left.\frac{1}{t} \cdot \frac{\partial u}{\partial n}\right|_{\partial \Omega}\right) \in\right.$ $\operatorname{ker} P\}$, for $\lambda \leq \lambda\left(t_{0}\right), t \in\left[t_{0}, 1\right]$.

Now the desired curve $\gamma$ in the Fredholm Lagrangian Grassmannian $F \Lambda_{\mu}(H)$ can then be defined by

$$
\gamma:(0,1] \rightarrow F \Lambda_{\mu}(H), \quad \gamma(t) \stackrel{\text { def }}{=} \tilde{\Phi}_{t} \circ T_{t}\left(K_{0, t}\right), t \in(0,1] .
$$

This curve describes the evolution of Fredholm Lagrangian subspaces of weak solutions for (2.3) as $t$ increases, where $\lambda=0$ is fixed. Notice that by Proposition 4.3 and Lemma 4.6 the curve $\gamma$ is a $C^{2}$ curve, and $\gamma(t)=W^{u}(0, t)$, for $t \in(0,1]$.

\section{Proof of the MAin Results}

In this section we present the proofs of Theorems 2.4, 2.5 and Corollaries 2.6, 2.7. Let the assumptions (Ha) be satisfied, and assume that $\bar{u} \in C^{2}(\bar{\Omega})$. We will first develop a method of calculating the Morse index $M(\bar{u})$ in terms of the Maslov index for the curve $\left.W^{u}(\cdot, 1)\right|_{[\lambda, 0]}$ for $\lambda<0$ sufficiently negative. This step will be common for the proofs of both Theorems 2.4 and 2.5.

By definition $M(\bar{u})$ is the dimension of the maximal subspace of

$$
H_{P}=\left\{w \in H^{2}(\Omega) \mid P\left(w, \frac{\partial w}{\partial n}\right)=0\right\}
$$

on which $A=-\triangle+f(x)$ is negative-definite. It therefore is the number of negative eigenvalues $\lambda_{i}$, counting with algebraic multiplicity, for the problem

$$
\begin{array}{r}
-\triangle w+f(x) w=\lambda w, \lambda<0, \\
P\left(\left.w\right|_{\partial \Omega},\left.\frac{\partial v}{\partial n}\right|_{\partial \Omega}\right)=0 .
\end{array}
$$

Let $d(\lambda)$ denote the dimension of the solution sets for (5.1), (5.2) if $\lambda$ is an eigenvalue, $d(\lambda)$ is then the geometric multiplicity of $\lambda$; also $d(\lambda)=\operatorname{dim}\left(W^{u}(\lambda, 1) \cap\right.$ $\mu)$. Since $A$ is selfadjoint when restricted to $H_{P}$, the algebraic multiplicity of $\lambda$ is the same as the geometric multiplicity of $\lambda$. We thus have

$$
M(\bar{u})=\sum_{\lambda<0} d(\lambda) .
$$

Also from the proof of Lemma 4.9 we know that $A-\lambda$ is positive definite on $H_{P}$ when $\lambda \leq \lambda(1)$; thus $\lambda$ will not be an eigenvalue for $A$ on $H_{P}$ if $\lambda \leq \lambda(1)$. We then have

$$
M(\bar{u})=\sum_{\lambda(1)<\lambda<0} d(\lambda)
$$


Lemma 5.1. The Morse index

$$
M(\bar{u})=-\mathbf{M} a s\left(\left.\{\gamma(t)\}\right|_{\left[t_{0}, 1\right]}, \mu\right)-\mathbf{M} a s\left(\left\{\left.W^{u}\left(\cdot, t_{0}\right)\right|_{\left[\lambda\left(t_{0}\right), 0\right]}\right\}, \mu\right)
$$

for any $t_{0} \in(0,1]$.

Proof. By Lemma 4.7, $W^{u}(\cdot, 1):(-\infty, 0] \rightarrow F \Lambda_{\mu}(H)$ is a negative curve in $F \Lambda_{\mu}(H)$, and from the proof of Lemma 4.9 we have that $W^{u}(\lambda, 1) \notin \mathcal{M}_{\mu}(H)$ for $\lambda \in\left(-\infty, \lambda\left(t_{0}\right)\right], t_{0} \in(0,1]$. Thus by Theorem 3.6 and Corollary 3.9 of section 3 , for any $t_{0} \in(0,1]$ we have

$$
\begin{array}{r}
-\mathbf{M} a s\left(\left\{\left.W^{u}(\cdot, 1)\right|_{\left[\lambda\left(t_{0}\right), 0\right]}\right\}, \mu\right)=-\mathbf{M} \operatorname{as}\left(\left\{\left.W^{u}(\cdot, 1)\right|_{[\lambda(1), 0]}\right\}, \mu\right) \\
=\sum_{\lambda(1)<s<0} \operatorname{dim}\left(W^{u}(s, 1) \cap \mu\right)=\sum_{\lambda(1)<s<0} d(s)=M(\bar{u}) .
\end{array}
$$

On the other hand, from the property (3) of the Maslov index as in Theorem 3.6 of section 3 , we have that for any $t_{0} \in(0,1]$,

$$
\begin{array}{r}
\mathbf{M} a s\left(\left\{\left.W^{u}\left(\cdot, t_{0}\right)\right|_{\left[\lambda\left(t_{0}\right), 0\right]}\right\}, \mu\right)+\mathbf{M} a s\left(\left\{\left.W^{u}(0, \cdot)\right|_{\left[t_{0}, 1\right]}\right\}, \mu\right) \\
=\mathbf{M} a s\left(\left\{\left.W^{u}\left(\lambda\left(t_{0}\right), \cdot\right)\right|_{\left[t_{0}, 1\right]}\right\}, \mu\right)+\mathbf{M} \operatorname{as}\left(\left\{\left.W^{u}(\cdot, 1)\right|_{\left[\lambda\left(t_{0}\right), 0\right]}\right\}, \mu\right) .
\end{array}
$$

Also we have

$$
\begin{aligned}
\mathbf{M} a s\left(\left\{\left.W^{u}\left(\lambda\left(t_{0}\right), \cdot\right)\right|_{\left[t_{0}, 1\right]}\right\}, \mu\right) & =0, \mathbf{M} a s\left(\left\{\left.W^{u}(0, \cdot)\right|_{\left[t_{0}, 1\right]}\right\}, \mu\right) \\
& =\mathbf{M} \operatorname{as}\left(\left.\{\gamma(t)\}\right|_{\left[t_{0}, 1\right]}, \mu\right),
\end{aligned}
$$

due to Lemma 4.9 and the definition of $\gamma$. Combining (5.5), (5.6) and (5.7) we get the following:

$$
M(\bar{u})=-\mathbf{M} a s\left(\left.\{\gamma(t)\}\right|_{\left[t_{0}, 1\right]}, \mu\right)-\mathbf{M} a s\left(\left\{\left.W^{u}\left(\cdot, t_{0}\right)\right|_{\left[\lambda\left(t_{0}\right), 0\right]}\right\}, \mu\right) .
$$

Remark 5.2. The argument above also applies to $A_{\lambda, t}=-\triangle+t^{2}(f(t x)-\lambda)$ on $H_{P, t}=\left\{u \in H^{2}(\Omega) \mid\left(\left.u\right|_{\partial \Omega},\left.\frac{1}{t} \cdot \frac{\partial u}{\partial n}\right|_{\partial \Omega}\right) \in \operatorname{ker} P\right\}$; in particular, the Morse index of $A_{0, t}=-\triangle+t^{2} f(t x)$ on $H_{P, t}$ is given by $-\mathbf{M} a s\left(\left\{\left.W^{u}(\cdot, t)\right|_{[\lambda(t), 0]}\right\}, \mu\right)$ for any $t \in(0,1]$.

Equation (5.8) gives a way of calculating the Morse index of $\bar{u}$ in terms of the Maslov index $\mathbf{M} a s\left(\left.\{\gamma(t)\}\right|_{\left[t_{0}, 1\right]}, \mu\right)$ with a correction term $\mathbf{M} a s\left(\left\{\left.W^{u}\left(\cdot, t_{0}\right)\right|_{\left[\lambda\left(t_{0}\right), 0\right]}\right\}\right.$, $\mu)$.

Proof of Theorem 2.4. By assumption we have ker $P=\left\{\left(S^{\prime} v, v\right) \mid v \in H^{-\frac{1}{2}}(\partial \Omega)\right\}$, where $S^{\prime}: H^{-\frac{1}{2}}(\partial \Omega) \rightarrow H^{\frac{1}{2}}(\partial \Omega)$ is compact, and $S^{\prime *}=S^{\prime}, S^{\prime} \leq 0$. For $t \in$ $(0,1], \gamma(t) \notin \mathcal{M}_{\mu}(H)$ if and only if

$$
\begin{array}{r}
-\triangle w+t^{2} f(t x) w=0, \quad x \in \Omega, \\
P\left(\left.w\right|_{\partial \Omega},\left.\frac{1}{t} \cdot \frac{\partial w}{\partial n}\right|_{\partial \Omega}\right)=0
\end{array}
$$

has no weak solution $w \in H^{1}(\Omega)$, which is equivalent to

$$
\begin{array}{r}
-\Delta w+t^{2} f(t x) w=0, \quad x \in \Omega, \\
\left.w\right|_{\partial \Omega}-\frac{1}{t} \cdot S^{\prime}\left(\left.\frac{\partial w}{\partial n}\right|_{\partial \Omega}\right)=0
\end{array}
$$


having no weak solution $w \in H^{1}(\Omega)$. Since $f(x)=g_{u}(x, \bar{u}(x)) \in C^{2}(\bar{\Omega})$, and $f(0)>0$, there exists $t_{0}>0$ such that $f(t x)>\frac{f(0)}{2}$ for $t \in\left[0, t_{0}\right], x \in \Omega$. Now for $t \in\left(0, t_{0}\right], u \in\left\{u \in H^{2}(\Omega)|u|_{\partial \Omega}-\frac{1}{t} \cdot S^{\prime}\left(\left.\frac{\partial u}{\partial n}\right|_{\partial \Omega}\right)=0\right\}$, we have

$$
\begin{aligned}
\left(\left(-\triangle+t^{2} f(t x)\right) u, u\right)_{L^{2}(\Omega)} & =-\int_{\partial \Omega} \frac{\partial u}{\partial n} u d y+\int_{\Omega}\left(|\nabla u|^{2}+t^{2} f(t x) u^{2}\right) d x \\
& =-\frac{1}{t} \cdot\left\langle S^{\prime}\left(\frac{\partial u}{\partial n} \mid \partial \Omega\right), \frac{\partial u}{\partial n} \mid \partial \Omega\right\rangle+\int_{\Omega}\left(|\nabla u|^{2}+t^{2} f(t x) u^{2}\right) d x \\
& >\int_{\Omega}\left(|\nabla u|^{2}+\frac{f(0)}{2} t^{2} u^{2} d x,\right.
\end{aligned}
$$

which shows that $A_{0, t}=-\triangle+t^{2} f(t x)$ is positive definite on $H_{P, t}=\left\{u \in H^{2}(\Omega) \mid\right.$ $\left.\left.u\right|_{\partial \Omega}-\frac{1}{t} \cdot S^{\prime}\left(\left.\frac{\partial u}{\partial n}\right|_{\partial \Omega}\right)=0\right\}$, for $t \in\left(0, t_{0}\right]$. Now by Remark 5.2, the Morse index of $A_{0, t}$ on $H_{P, t}$, which is zero, is the same as $-\mathbf{M} a s\left(\left\{\left.W^{u}(\cdot, t)\right|_{[\lambda(t), 0]}\right\}, \mu\right)$. Therefore from Equation (5.8) we get that for $t \in\left(0, t_{0}\right]$,

$$
M(\bar{u})=-\mathbf{M} a s\left(\left.\{\gamma(\cdot)\}\right|_{[t, 1]}, \mu\right) .
$$

Thus we have completed the proof of Theorem 2.4.

Proposition 5.3. Let $\operatorname{ker} P$ be a Neumann-based Lagrangian subspace of $H$. If we have $\left\langle\left. 1\right|_{\partial \Omega}, S\left(\left.1\right|_{\partial \Omega}\right)\right\rangle \quad\left\langle 0\right.$, or $\left\langle\left. 1\right|_{\partial \Omega}, S\left(\left.1\right|_{\partial \Omega}\right)\right\rangle=0, f(0)>0$, then there exists $t_{0}>0$, such that for $t \in\left(0, t_{0}\right]$, we have $\gamma(t) \notin \mathcal{M}_{\mu}(H)$ and $A_{0, t}=-\triangle+t^{2} f(t x)$ is positive definite on $H_{P, t}$.

Proof. By assumption we have $\mu=\operatorname{ker} P=\left\{(u, S u) \mid u \in H^{\frac{1}{2}}(\partial \Omega)\right\}$, where $S$ : $H^{\frac{1}{2}}(\partial \Omega) \rightarrow H^{-\frac{1}{2}}(\partial \Omega)$ is compact, and $S^{*}=S$. For $t \in(0,1], \gamma(t) \notin \mathcal{M}_{\mu}(H)$ if and only if

$$
\begin{array}{r}
-\triangle w+t^{2} f(t x) w=0, \quad x \in \Omega \\
P\left(\left.w\right|_{\partial \Omega},\left.\frac{1}{t} \cdot \frac{\partial w}{\partial n}\right|_{\partial \Omega}\right)=0
\end{array}
$$

has no weak solution $w \in H^{1}(\Omega)$, which is equivalent to

$$
\begin{array}{r}
-\triangle w+t^{2} f(t x) w=0, \quad x \in \Omega, \\
\left.\frac{\partial w}{\partial n}\right|_{\partial \Omega}=t S\left(\left.w\right|_{\partial \Omega}\right)
\end{array}
$$

having no weak solution $w \in H^{1}(\Omega)$. Denoting $L_{N}=\left\{(u, 0) \mid u \in H^{\frac{1}{2}}(\partial \Omega)\right\}$, we have that

$$
\xi(t) \stackrel{\text { def }}{=}\left\{\left(\left.w\right|_{\partial \Omega},\left.\frac{\partial w}{\partial n}\right|_{\partial \Omega}-t S\left(\left.w\right|_{\partial \Omega}\right)\right) \mid-\triangle w+t^{2} f(t x) w=0, w \in H^{1}(\Omega)\right\}
$$

is a $C^{2}$ curve in $F \Lambda_{L_{N}}(H)$, for $t \in[0,1]$. Notice that $\xi(0) \cap L_{N}=\left\{c \cdot\left(\left.1\right|_{\partial \Omega}, 0\right) \mid c \in R\right\}$, and $\gamma(t) \notin \mathcal{M}_{\mu}(H)$ if and only if $\xi(t) \notin \mathcal{M}_{L_{N}}(H)$ for $t \in(0,1]$.

We now calculate the crossing form for $\xi(t) \in F \Lambda_{L_{N}}(H)$ at $t=0$. Let $\nu=$ $\left\{(0, v) \mid v \in H^{-\frac{1}{2}}(\partial \Omega)\right\}$. Then $\xi(t)$ is transversal to $\nu$ for sufficiently small $t \ll 1$. Therefore there exists a $C^{2}$ differentiable family of bounded operators $\left\{\phi_{t}\right\}_{t \ll 1}, \phi_{t}$ : $L_{N} \rightarrow \nu$, so that $\xi(t)=$ graph of $\phi_{t}$, for $t \ll 1$.

Fix $q=\left(\left.c\right|_{\partial \Omega}, 0\right) \in \xi(0) \cap L_{N}, c \neq 0$. We consider the curve

$$
X(t)=q+\phi_{t}(q) \in \xi(t), t \ll 1 .
$$


By definition of $\xi(t)$ there exists a $C^{2}$ curve of solutions $w(t)$ for

$$
-\triangle w+t^{2} f(t x) w=0
$$

such that $\left(\left.w(t)\right|_{\partial \Omega},\left.\frac{\partial w(t)}{\partial n}\right|_{\partial \Omega}-t S\left(\left.w(t)\right|_{\partial \Omega}\right)\right) \in H$ is exactly $X(t)$. We then have the following.

Claim 5.4. $\omega\left(X(0), \frac{d X}{d t}(0)\right)=-c^{2}\left\langle\left. 1\right|_{\partial \Omega}, S\left(\left.1\right|_{\partial \Omega}\right)\right\rangle$.

Proof of Claim 5.4. We have

$$
\omega\left(X, \frac{d X}{d t}\right)=\int_{\partial \Omega} w \frac{\partial^{2} w}{\partial t \partial n}-\frac{\partial w}{\partial t} \cdot \frac{\partial w}{\partial n}+\left\langle w,-S w-t S\left(\frac{\partial w}{\partial t}\right)\right\rangle+t\left\langle\frac{\partial w}{\partial t}, S(w)\right\rangle d y
$$

whereas, from (5.19) we have

$$
\left(-\triangle+t^{2} f(t x)\right) \frac{\partial w}{\partial t}+2 t f(t x) w+t^{2}(\nabla f(t x) \cdot x) w=0 .
$$

By multiplying (5.21) with $w$ and integrating over $\Omega$, we have

$$
\begin{aligned}
\int_{\partial \Omega} w \frac{\partial^{2} w}{\partial t \partial n} d y= & \iint_{\Omega} \nabla w \cdot \nabla\left(\frac{\partial w}{\partial t}\right)+\frac{\partial w}{\partial t} \cdot\left(t^{2} f(t x)\right) w+\left(t^{2}(\nabla f(t x) \cdot x)+2 t f(t x)\right) w^{2} d x \\
= & \int_{\partial \Omega} \frac{\partial w}{\partial n} \cdot \frac{\partial w}{\partial t} d y \\
& \quad+\iint_{\Omega} \frac{\partial w}{\partial t} \cdot\left(-\triangle+t^{2} f(t x)\right) w+\left(t^{2}(\nabla f(t x) \cdot x)+2 t f(t x)\right) w^{2} d x
\end{aligned}
$$

Combining (5.20) and (5.22) we have

$$
\omega\left(X(0), \frac{d X}{d t}(0)\right)=\left\langle\left. c\right|_{\partial \Omega},-S\left(\left.c\right|_{\partial \Omega}\right)\right\rangle=-c^{2} \cdot\left\langle\left. 1\right|_{\partial \Omega}, S\left(\left.1\right|_{\partial \Omega}\right)\right\rangle .
$$

Therefore the proof of Claim 5.4 is complete.

For each $q=\left(\left.c\right|_{\partial \Omega}, 0\right) \in \xi(0) \cap L_{N}, c \neq 0$, from Claim 5.4 we have

$$
\left.Q_{\mathcal{M}}(q, q) \stackrel{\text { def }}{=} \frac{d}{d t}\right|_{t=0} \omega\left(q, q+\phi_{t}(q)\right)=\omega\left(X(0), \frac{\partial X}{\partial t}(0)\right)=-c^{2} \cdot\left\langle\left. 1\right|_{\partial \Omega}, S\left(\left.1\right|_{\partial \Omega}\right)\right\rangle ;
$$

thus when the condition $\left\langle\left. 1\right|_{\partial \Omega}, S\left(\left.1\right|_{\partial \Omega}\right)\right\rangle<0$ is satisfied, it follows that $t=0$ is a positive crossing for the curve $\xi(t)$. Therefore $t=0$ is an isolated crossing point for $\xi(t)$ in $F \Lambda_{L_{N}}(H)$; i.e., there exists $t_{0}>0$ such that $\xi(t) \notin \mathcal{M}_{L_{N}}(H)$ for $t \in\left(0, t_{0}\right]$. Necessarily we have $\gamma(t) \notin \mathcal{M}_{\mu}(H)$ for $t \in\left(0, t_{0}\right]$.

To show that $A_{0, t}=-\triangle+t^{2} f(t x)$ is positive definite on $H_{P, t}$ for $t \in\left(0, t_{0}\right]$, if $\left\langle\left. 1\right|_{\partial \Omega}, S\left(\left.1\right|_{\partial \Omega}\right)\right\rangle \quad<0$, we note that $\phi_{t}: L_{N} \rightarrow \nu$ satisfies $\phi_{t}^{*}=\phi_{t}$, and $\phi_{0}=\mathbf{N}$, the standard Dirichlet-to-Neumann operator. Now the following lemma (see Kato [15) together with the calculation of the crossing form, shows that $\phi_{t}$ is positive definite for $t>0$ sufficiently small, since now the simple eigenvalue zero of $\phi_{0}=\mathbf{N}$ will move to the positive real part due to Lemma 5.5, when $t>0$ is turned on.

Lemma 5.5. Let $\left\{A_{t}\right\}_{|t| \ll 1}$ be a $C^{1}$-class family of selfadjoint Fredholm operators on a Hilbert space $H$. Assume that the symmetric bilinear form on $\operatorname{ker}\left(A_{0}\right)$,

$$
Q(x, y)=\frac{d}{d t}\left(x, A_{t}(y)\right)_{\mid t=0}=\left(x, \dot{A}_{0}(y)\right), x, y \in \operatorname{ker}\left(A_{0}\right),
$$

is nondegenerate. Then there exists a positive number $\delta$ such that for $0<t \leq \delta$, there exist $p$ positive eigenvalues and $q$ negative eigenvalues of the operator $A_{t}$, 
where $p-q=\operatorname{sign} Q, p+q=\operatorname{dim} \operatorname{ker}\left(A_{0}\right)$. Also for $-\delta \leq t<0$ the oppositive situations hold.

Since $A_{0, t}=-\triangle+t^{2} f(t x)$ being positive definite on $H_{P, t}$ for $t \in\left(0, t_{0}\right]$ is equivalent to $\phi_{t}$ being positive definite, we have that there exists $t_{0}>0$, such that $A_{0, t}$ is positive definite on $H_{P, t}$ for $t \in\left(0, t_{0}\right]$.

In the second case of ker $P=\left\{(u, S u) \mid u \in H^{\frac{1}{2}}(\partial \Omega)\right\}$, where $S: H^{\frac{1}{2}}(\partial \Omega) \rightarrow$ $H^{-\frac{1}{2}}(\partial \Omega)$ is compact, and $S^{*}=S,\left\langle\left. 1\right|_{\partial \Omega}, S\left(\left.1\right|_{\partial \Omega}\right)\right\rangle=0, f(0)>0$, the crossing form on $\xi(0) \cap L_{N}$,

$$
\left.Q_{\mathcal{M}}(q, q) \stackrel{\text { def }}{=} \frac{d}{d t}\right|_{t=0} \omega\left(q, q+\phi_{t}(q)\right)=-c^{2} \cdot\left\langle\left. 1\right|_{\partial \Omega}, S\left(\left.1\right|_{\partial \Omega}\right)\right\rangle \equiv 0,
$$

will vanish, for $q=\left(\left.c\right|_{\partial \Omega}, 0\right) \in \xi(0) \cap L_{N}$, while the secondary crossing form is given by

$$
\left.Q_{\mathcal{M}}^{(2)}(q, q) \stackrel{\text { def }}{=} \frac{d^{2}}{d t^{2}}\right|_{t=0} \omega\left(q, q+\phi_{t}(q)\right)=\iint_{\Omega} 2 f(0) w(0)^{2} d x=2 f(0) c^{2}>0
$$

for $q=\left(\left.c\right|_{\partial \Omega}, 0\right) \in \xi(0) \cap L_{N}, c \neq 0$, under the condition that $f(0)>0$. Therefore $t=0$ is an isolated crossing point for $\xi(t)$ in $F \Lambda_{L_{N}}(H)$.

Now by using the following analog of Lemma 5.5, together with Equation (5.24) above, it follows that $\phi_{t}$ is positive definite for $t>0$ sufficiently small.

Lemma 5.6. Let $\left\{A_{t}\right\}_{|t| \ll 1}$ be a $C^{2}$-class family of selfadjoint Fredholm operators on a Hilbert space $H$. Assume that the symmetric bilinear form on $\operatorname{ker}\left(A_{0}\right)$,

$$
Q(x, y)=\frac{d}{d t}\left(x, A_{t}(y)\right)_{\mid t=0} \equiv 0, \quad Q^{(2)}(x, y)=\frac{d^{2}}{d t^{2}}\left(x, A_{t}(y)\right)_{\mid t=0}, x, y \in \operatorname{ker}\left(A_{0}\right),
$$

is nondegenerate. Then there exists a positive number $\delta$, such that for $0<t \leq \delta$, there exist $p$ positive eigenvalues and $q$ negative eigenvalues of the operator $A_{t}$, where $p-q=\operatorname{sign} Q^{(2)}, p+q=\operatorname{dim} \operatorname{ker}\left(A_{0}\right)$. Also for $-\delta \leq t<0$ the same situations hold.

This thus leads to the statement that $A_{0, t}=-\triangle+t^{2} f(t x)$ is positive definite on $H_{P, t}$ for $t \in\left(0, t_{0}\right]$, some $t_{0}>0$, if $\left\langle\left. 1\right|_{\partial \Omega}, S\left(\left.1\right|_{\partial \Omega}\right)\right\rangle=0, f(0)>0$.

Corollary 5.7. Let $\operatorname{ker} P$ be a Neumann-based Lagrangian subspace of $H$. If we have $\left\langle\left. 1\right|_{\partial \Omega}, S\left(\left.1\right|_{\partial \Omega}\right)\right\rangle>0$, or $\left\langle\left. 1\right|_{\partial \Omega}, S\left(\left.1\right|_{\partial \Omega}\right)\right\rangle=0, \quad f(0)<0$, then $\gamma(t) \notin$ $\mathcal{M}_{\mu}(H)$, and $A_{0, t}=-\triangle+t^{2} f(t x)$ is of Morse index 1 on $H_{P, t}$ for $t \in\left(0, t_{0}\right]$, some $t_{0}>0$.

Proof of Theorem 2.5. Combining equation (5.8) and Remark 5.2 we have that

$$
M(\bar{u})=-\mathbf{M} a s\left(\left.\{\gamma(\cdot)\}\right|_{[t, 1]}, \mu\right)+\text { Morse index of } A_{0, t} \text { on } H_{P, t},
$$

for any $t \in(0,1]$. Theorem 2.5 then follows from Proposition 5.3 and Corollary 5.7 .

Proof of Corollary 2.6. Let (Ha) be satisfied. Let $\bar{u} \in C^{1}(\bar{\Omega})$ be a solution for (2.1) and (2.2). First we show that if $\mu=L_{D} \stackrel{\text { def }}{=}\left\{(0, v) \mid v \in H^{-\frac{1}{2}}(\partial \Omega)\right\}$, then we have

Claim 5.8. $\{\gamma(t)\}_{t \in(0,1]}$ is a $C^{1}$ negative curve in $F \Lambda_{\mu}(H)$, and there exists $t_{0}>0$ such that $\gamma(t) \notin \mathcal{M}_{\mu}(H)$ for $t \in\left(0, t_{0}\right]$. 
Proof of Claim 5.8. The statement that $\{\gamma(t)\}_{t \in(0,1]}$ is a $C^{1}$ curve in $F \Lambda_{\mu}(H)$ follows from Remark 4.2 and Proposition 4.3 of section 4, by replacing $u \in C^{2}(\bar{\Omega})$ by $u \in C^{1}(\bar{\Omega})$ and correspondingly showing that $\gamma$ is a $C^{1}$ curve. To show that $\gamma(t) \notin \mathcal{M}_{\mu}(H)$ for $t \in\left(0, t_{0}\right]$, some $t_{0}>0$, notice that for $t \in(0,1], \gamma(t) \notin \mathcal{M}_{\mu}(H)$ if and only if

$$
\begin{array}{r}
-\triangle w+t^{2} f(t x) w=0, \quad x \in \Omega, \\
P\left(\left.w\right|_{\partial \Omega},\left.\frac{1}{t} \cdot \frac{\partial w}{\partial n}\right|_{\partial \Omega}\right)=0
\end{array}
$$

has no weak solution $w \in H^{1}(\Omega)$, which is equivalent to

$$
\begin{array}{r}
-\triangle w+t^{2} f(t x) w=0, \quad x \in \Omega, \\
\left.w\right|_{\partial \Omega}=0
\end{array}
$$

having no weak solution $w \in H^{1}(\Omega)$. Since $-\triangle$ is positive definite on $\left\{u \in H^{2}(\Omega)\right.$ $\left.|u|_{\partial \Omega}=0\right\}, A_{0, t}=-\triangle+t^{2} f(t x)$ is positive definite on $\left\{u \in H^{2}(\Omega)|u|_{\partial \Omega}=0\right\}$, for $t \in\left(0, t_{0}\right]$, some $t_{0}>0$; consequently there exists no $H^{1}$ weak solution $w$ for (5.27) and (5.28), which is equivalent to $\gamma(t) \notin \mathcal{M}_{\mu}(H)$ for $t \in\left(0, t_{0}\right]$.

To show that $\gamma$ is a negative curve, let $t^{*} \in(0,1]$ be a crossing of $\gamma(t)$, i.e., $\gamma\left(t^{*}\right) \cap \mu \neq\{0\}$. It suffices to show that $t=t^{*}$ is a negative crossing. Let $\nu$ be a Lagrangian subspace which is transversal to $\gamma\left(t^{*}\right)$. Then $\gamma(t)$ is transversal to $\nu$ for sufficiently small $\left|t-t^{*}\right| \ll 1$. Therefore there exists a $C^{1}$ differentiable family of bounded operators $\left\{\phi_{t}\right\}_{\left|t-t^{*}\right| \ll 1}, \phi_{t}: \gamma\left(t^{*}\right) \rightarrow \nu$, so that $\gamma(t)=$ graph of $\phi_{t}$, for $\left|t-t^{*}\right| \ll 1$.

Fixing $q \in \gamma\left(t^{*}\right) \cap \mu, q \neq 0$, by definition there exists a solution $\hat{w}(x)$ for

$$
-\triangle w+\left(t^{*}\right)^{2} f\left(t^{*} x\right) w=0,
$$

such that the trace map $\left(\left.\hat{w}\right|_{\partial \Omega},\left.\frac{1}{t^{*}} \cdot \frac{\partial \hat{w}}{\partial n}\right|_{\partial \Omega}\right)=\left(\left.0\right|_{\partial \Omega},\left.\frac{1}{t^{*}} \cdot \frac{\partial \hat{w}}{\partial n}\right|_{\partial \Omega}\right) \in H$ is exactly $q$. Then for $\left|t-t^{*}\right| \ll 1, w(t)=\hat{w}\left(\frac{t x}{t^{*}}\right)$ gives a $C^{1}$ curve of solutions for

$$
-\triangle w+t^{2} f(t x) w=0,
$$

and we define the trace map $\left(\left.w(t)\right|_{\partial \Omega},\left.\frac{1}{t} \cdot \frac{\partial w(t)}{\partial n}\right|_{\partial \Omega}\right) \in H$ to be $X(t)$. In this way, $X(t) \in \gamma(t), X\left(t^{*}\right)=q$. Thus we have $X(t)=a(t)+b(t)$, where $a(t) \in \gamma\left(t^{*}\right), b(t) \in$ $\nu$, and $b(t)=\phi(t)(a(t)), b\left(t^{*}\right)=\phi\left(t^{*}\right)\left(a\left(t^{*}\right)\right)=\phi\left(t^{*}\right)(q)=0$. It follows that

$$
\omega\left(X\left(t^{*}\right), \frac{d X}{d t}\left(t^{*}\right)\right)=\omega\left(q, \dot{a}\left(t^{*}\right)\right)+\phi\left(t^{*}\right)\left(\dot{a}\left(t^{*}\right)\right)+\dot{\phi}\left(t^{*}\right)\left(a\left(t^{*}\right)\right)=\omega\left(q, \dot{\phi}\left(t^{*}\right)(q)\right),
$$

where in the second equality we used the fact that both $q$ and $\dot{a}\left(t^{*}\right)+\phi\left(t^{*}\right)\left(\dot{a}\left(t^{*}\right)\right)$ belong to $\gamma\left(t^{*}\right)$, and $\omega$ vanishes on $\gamma\left(t^{*}\right)$. The crossing form

$$
\begin{array}{r}
\left.Q_{\mathcal{M}}(q, q) \stackrel{\text { def }}{=} \frac{d}{d t}\right|_{t=t^{*}} \omega\left(q, \phi_{t}(q)\right)=\omega\left(X\left(t^{*}\right), \frac{d X}{d t}\left(t^{*}\right)\right) \\
=\frac{1}{t^{*}} \int_{\partial \Omega}-\frac{\partial w}{\partial n} \cdot \frac{\partial w}{\partial t} d y=\frac{1}{\left(t^{*}\right)^{2}} \int_{\partial \Omega}-\left(\frac{\partial w}{\partial n}\right)^{2} \cdot(y \cdot n) d y<0,
\end{array}
$$

for $q \in \gamma\left(t^{*}\right) \cap \mu$; i.e., $Q_{\mathcal{M}}$ is negative definite on $\gamma\left(t^{*}\right) \cap \mu$. Thus by definition $t=t^{*}$ is a negative crossing for the curve $\gamma(t)$. The proof of Claim 5.8 is complete.

Since $\gamma$ is a negative curve in $F \Lambda_{\mu}(H)$, and there exists $t_{0}>0$ such that $\gamma(t) \notin$ $\mathcal{M}_{\mu}(H)$ for $t \in\left(0, t_{0}\right]$, by Corollary 3.9 we have

$$
\mathbf{M} a s\left(\left\{\left.\gamma(\cdot)\right|_{[t, 1]}\right\}, \mu\right)=-\sum_{t \leq t_{i}<1} \operatorname{dim}\left(\gamma\left(t_{i}\right) \cap \mu\right)=-\sum_{t<t_{i}<1} \operatorname{dim}\left(\gamma\left(t_{i}\right) \cap \mu\right)
$$


for $t \in\left(0, t_{0}\right]$. Now from equation (5.8) we also have

(5.34)

$M(\bar{u})=-\mathbf{M} a s\left(\left.\{\gamma(\cdot)\}\right|_{[t, 1]}, \mu\right)-\mathbf{M} a s\left(\left\{\left.W^{u}(\cdot, t)\right|_{[\lambda(t), 0]}\right\}, \mu\right)=-\mathbf{M} a s\left(\left.\{\gamma(\cdot)\}\right|_{[t, 1]}, \mu\right)$,

for $t \in\left(0, t_{0}\right]$, where the last equality then follows by observing that

$$
-\mathbf{M} a s\left(\left\{\left.W^{u}(\cdot, t)\right|_{[\lambda(t), 0]}\right\}, \mu\right)
$$

is the same as the Morse index of $A_{0, t}=-\triangle+t^{2} f(t x)$ on $\left\{u \in H^{2}(\Omega)|u|_{\partial \Omega}=0\right\}$, which is zero if $t_{0}$ is sufficiently small.

Combining (5.33) and (5.34) we get

$$
M(\bar{u})=\sum_{t<t_{i}<1} \operatorname{dim}\left(\gamma\left(t_{i}\right) \cap \mu\right)=\sum_{0<t_{i}<1} \operatorname{dim}\left(\gamma\left(t_{i}\right) \cap \mu\right) .
$$

Thus we complete the proof of Corollary 2.6.

Proof of Corollary 2.7. Let (Ha) be satisfied. Let $\bar{u} \in C^{2}(\bar{\Omega})$ be a solution for (2.1) and (2.2). By assumption we have $\mu=L_{N}=\left\{(u, 0) \mid u \in H^{\frac{1}{2}}(\partial \Omega)\right\}$, and $t=1$ is a crossing of $\gamma(t)$ with $\mu$, with $\operatorname{dim}(\gamma(1) \cap \mu)=1$. For any $q \in \gamma(1) \cap \mu, q \neq 0$, by definition there exists $c \neq 0$, such that the trace map $\left(\left.c \cdot \hat{w}\right|_{\partial \Omega},\left.c \cdot \frac{\partial \hat{w}}{\partial n}\right|_{\partial \Omega}\right)=$ $\left(\left.c \cdot \hat{w}\right|_{\partial \Omega},\left.0\right|_{\partial \Omega}\right) \in H$ is exactly $q$. Then for $0 \leq 1-t \ll 1, w(t)=c \cdot \hat{w}(t x)$ gives a $C^{1}$ curve of solutions for

$$
-\triangle w+t^{2} f(t x) w=0
$$

and we define the trace map $\left(\left.w(t)\right|_{\partial \Omega},\left.\frac{1}{t} \cdot \frac{\partial w(t)}{\partial n}\right|_{\partial \Omega}\right) \in H$ to be $X(t)$. A calculation of the crossing form similar to that for Corollary 2.6 gives

$$
Q_{\mathcal{M}}(q, q)=c^{2} \cdot \int_{\partial \Omega} \bar{w} \cdot\left[n \cdot \nabla_{x}\left(x \cdot \nabla_{x} \bar{w}\right)\right] d y>0 ;
$$

thus $t=1$ is a positive crossing. Thus by Theorem 2.5 and Proposition 3.8 we have

$$
M(1)-M(1-\delta)=-\mathbf{M} a s\left(\left.\{\gamma(t)\}\right|_{[1-\delta, 1]}, \mu\right)=-1
$$

for $\delta>0$ sufficiently small. Thus we have completed the proof of Corollary 2.7.

\section{ACKNOWLEDGMENT}

The research of the first author was supported in part by NSFC under grant \#10601014, and the research of the second author was supported by the National Science Foundation under grant DMS-0410267.

\section{REFERENCES}

1. V.I. Arnol'd, Characteristic class entering into quantization conditions, Funct. Anal. Appl. 1, 1-14. (1967) MR0211415 (35:2296)

2. V.I. Arnol'd, The Sturm theorem and symplectic geometry, Funct. Anal. Appl. 19, 251-259. (1985) MR:820079 (87j:58033)

3. R. Courant and D. Hilbert, Methods of mathematical physics, Vols. I, II. 1989. MR0065391 (16:426a) MR1013360 (90k:35001)

4. K. C. Chang, Infinite-dimensional Morse theory and multiple solution problems. Birkhäuser Boston, Inc., Boston, MA, 1993. MR1196690 (94e:58023)

5. S. E. Cappell, R. Lee and E. Y. Miller, On the Maslov Index, Comm. Pure. Appl. Math., Vol. XLVII, 121-186. (1994) MR1263126 (95f:57045)

6. S. E. Cappell, R. Lee and E. Y. Miller, Self-adjoint elliptic operators and manifold decompositions (II) Spectral flow and Maslov index, Comm. Pure. Appl. Math., Vol. XLIX, 869-909. (1996) MR1399200 (97g:58163) 
7. J. Deng, S. Nii, Infinite-dimensional Evans function theory for elliptic eigenvalue problems in a channel, J. Differential Equations 225 no. 1, 57-89. (2006) MR2228692 (2007b:35086)

8. J. Deng, S. Nii, An infinite dimensional Evans function theory for elliptic boudary value problems, Journal of Differential Equations 244 no. 4, 753-765. (2008) MR 2391343 (2009a:35180)

9. F. H. Lin, Nodal Set of Solutions of Elliptic and Parabolic Equations. Comm. Pure Appl. Math., 45, 287-308. (1991) MR1090434 (92b:58224)

10. D. Gilbarg, N. S. Trudinger, Elliptic Partial Differntial Equations of Second Order, Springer, Berlin, 2001. MR1814364 (2001k:35004)

11. P. Drábek, S. B. Robinson, On the Generalization of the Courant Nodal Domain Theorem, Journal of Differential Equations, Volume 181, Number 1, pp. 58-71(14) May 2002. MR.1900460 (2003g:35170)

12. R. Johnson, Oscillation theory and the density of states for the Schrödinger operator in odd dimension,, J. Diff. Equa., 92 no. 1, 145-162 (1991). MR.1113593 (93b:35033)

13. C.K.R.T. Jones, Instability of standing waves for non-linear Schrödinger-type equations, Ergod. Th. \& Dynam. Sys. 8, 119-138. (1988) MR967634 (90d:35267)

14. K. Furutani, Fredholm-Lagrangian-Grassmannian and the Maslov index, Journal of Geometry and Physics Volume 51, Issue 3, Pages 269-331 (2004) MR2079414 (2005g:53150)

15. T. Kato, Perturbation theory for linear operators, 2nd ed., Springer-Verlag, Berlin, 1980. MR 1335452 (96a:47025)

16. M. Struwe, Variational methods. Applications to nonlinear partial differential equations and Hamiltonian systems. Third edition. Springer-Verlag, Berlin, 2000. xviii +274 pp. MR.1736116 (2000i:49001)

17. J. Milnor, Morse Theory, Princeton University Press, 1964. MR.0163331 (29:634)

18. S. Smale, On the Morse index theorem, J. Math. Mech. 14, 1049-1056. (1965) MR0182027 $(31: 6251)$

19. R. C. Swanson, Fredholm intersection theory and elliptic boundary defqormation problems (I), (II), J. Differential Equations 28, 189-201, 202-219. (1978) MR491049 (80i:58024a) MR 0491050 (80i:58024b)

20. M. E. Taylor, Partial Differential Equations (II), Springer-Verlag, 1996. MR.1395149 (98b:35003)

21. W. Strauss, Partial Differential Equations, 1992. MR.1159712 (92m:35001)

22. X.F. Wang, W.-M. Ni, On the first positive Neumann eigenvalue, Discrete and Continuous Dynamical Systems 17, 1-19 (2007). MR.2257415 (2007h:35055)

Cema, Central University of Finance and Economics, Beijing, People's Republic of CHINA, 100085

E-mail address: jdeng@fudan.edu.cn

Department of Mathematics, University of North Carolina at Chapel Hill, Chapel Hill, North Carolina 27599 - And - Warwick Mathematics Institute, University of WarWiCK, UNiTED Kingdom

E-mail address: ckrtj@email.unc.edu 